\title{
Distributed Rate Allocation for Inelastic Flows
}

\author{
Prashanth Hande, Shengyu Zhang, and Mung Chiang, Member, IEEE
}

\begin{abstract}
A common assumption behind most of the recent research on network rate allocation is that traffic flows are elastic, which means that their utility functions are concave and continuous and that there is no hard limit on the rate allocated to each flow. These critical assumptions lead to the tractability of the analytic models for rate allocation based on network utility maximization, but also limit the applicability of the resulting rate allocation protocols. This paper focuses on inelastic flows and removes these restrictive and often invalid assumptions.

First, we consider nonconcave utility functions, which turn utility maximization into difficult, nonconvex optimization problems. We present conditions under which the standard price-based distributed algorithm can still converge to the globally optimal rate allocation despite nonconcavity of utility functions. In particular, continuity of price-based rate allocation at all the optimal prices is a sufficient condition for global convergence of rate allocation by the standard algorithm, and continuity at at least one optimal price is a necessary condition. We then show how to provision link capacity to guarantee convergence of the standard distributed algorithm. Second, we model real-time flow utilities as discontinuous functions. We show how link capacity can be provisioned to allow admission of all real-time flows, then propose a price-based admission control heuristics when such link capacity provisioning is impossible, and finally develop an optimal distributed algorithm to allocate rates between elastic and real-time flows.
\end{abstract}

Index Terms-Capacity provisioning, congestion control, inelastic flow, network control by pricing, network utility maximization, optimization, resource allocation.

\section{INTRODUCTION}

\section{A. Motivation and Organization}

I N A SEMINAL paper published in 1995, Shenker [18] discussed Internet service models to support applications beyond best-effort, in the framework of network utility models for four types of traffic. In particular, two major characteristics of 'elastic traffic' were highlighted: "These applications are rather elastic in nature, in that they tolerate packet delays and packet losses rather gracefully... Moreover, because of this elasticity, they can decrease their transmission rate in the presence of congestion." Utility functions for elastic traffic were modeled as smooth,

Manuscript received November 30, 2005; revised September 17, 2006; approved by IEEE/ACM TRANSACTIONS ON NETWORKING Editor F. Paganini. This work was supported in part by National Science Foundation (NSF) Grants CCF-0440443, CNS-0417607, and CNS-0427677, in part by the Air Force Office of Scientific Research (AFOSR) Grant FA9550-06-1-0297, in part by the Defense Research Advanced Projects Agency (DARPA) Grant HR0011-06-1-0008, and in part by the CBMANET program. Part of this paper was presented at IEEE INFOCOM 2005.

P. Hande is with the Electrical Engineering Department, Princeton University, Princeton, NJ 08542 USA and also with Qualcomm Flarion Technologies, Bedminster, NJ 07921 USA (e-mail: phande@ princeton.edu)

S. Zhang is with the Computer Science Department, California Institute of Technology, Pasadena, CA 91125 USA.

M. Chiang is with the Electrical Engineering Department, Princeton University, Princeton, NJ 08542 USA.

Digital Object Identifier 10.1109/TNET.2007.896507 concave functions of data rates, which lead to the conclusion in [18] that network utility is always maximized when no users are denied access. Over the last decade, maximization of concave utility functions and the resulting distributed rate allocation for elastic traffic have gained extensive attention. Elegant analytic results and rigorous mathematical frameworks for a standard price-based distributed algorithm become possible because of the concavity and continuity assumptions on utility functions and the elasticity assumption on application traffic. In particular, it has been shown (e.g., [12], [19]) that TCP congestion control can be reverse-engineered as implicitly solving concave utility maximization through price-based distributed subgradient algorithm.

However, it appears that the other classes of traffic (e.g., realtime, delay-adaptive, or streaming traffic) are not amenable to the utility maximization framework, and applicability of the distributed subgradient algorithm for rate allocation among these inelastic flows remains an open problem. Utility functions for inelastic flows are nonconcave or discontinuous, and they can only tolerate a limited amount of packet delay or fluctuations during rate allocation transients. Furthermore, when the source data rate requirements are rigid, some inelastic sources might be amenable only to admission control, which introduces booleanvalued variables and turn the problem into discrete optimization.

This paper tackles the difficulties of distributed rate allocation for inelastic flows. There are two standard ways to model inelastic traffic's utility function: a nonconcave utility function based on user perception model (e.g., sigmoidal utility for voice traffic based on MOS scores) or a discontinuous utility function based on the model where real-time flows attain zero utility if the rate is below a threshold and a constant positive utility (or concave increasing utility) above the threshold.

In the first part of the paper, Sections II and III, we examine the case of nonconcave utility functions. Recent work [11] has shown that the standard price-based distributed rate allocation may not even converge when one of the flows has a sigmoidal utility function. We answer the following question in Section II: "When will the standard distributed algorithm converge to the globally optimal rate allocation even when some flows have nonconcave utilities?" Then in Section III, we answer a further question: "Can link capacities be provisioned to guarantee such convergence?"

In the second part of the paper, Section IV, we examine the case of discontinuous utility functions for real-time traffic flows, and answer the following questions in sequence: "Can link capacities be provisioned such that all real-time flows can be admitted? If not, how should the admission control decision be distributively made based on pricing information? And how can a fair share of link capacities be ensured for elastic flows sharing links with real-time flows?"

An overview of the answers to the above questions will be presented in Section I-C, after a brief review of distributed rate allocation through concave and continuous utility maximization in Section I-B. 


\section{B. Review: Concave and Continuous Utility Maximization}

Since the publication of the seminal paper [9] by Kelly et al. in 1998, the framework of network utility maximization (NUM) has found many applications in network rate allocation algorithms and Internet congestion control protocols [5], in particular, distributed solutions of NUM are used to share link capacities among sources, and TCP congestion control variants are implicitly solving NUM for different utility functions (e.g., [10], [12], [14], [15], [19], [21]).

Consider a communication network with $L$ links, each with a fixed capacity of $c_{l}$ bps, and $S$ sources, each transmitting at a source rate of $x_{s}$ bps. Each logical source emits one flow (thus we will use the terms 'source' and 'flow' interchangeably), using a fixed set $L(s)$ of links in its path, and has a utility function $U_{s}\left(x_{s}\right)$. The basic version of NUM is the problem of maximizing the total network utility $\sum_{s} U_{s}\left(x_{s}\right)$, over the source rates $\mathbf{x}$, subject to linear flow feasibility constraints $\sum_{s: l \in L(s)} x_{s} \leq c_{l}$ for all links $l$

$$
\begin{array}{ll}
\text { maximize } & \sum_{s} U_{s}\left(x_{s}\right) \\
\text { subject to } & \sum_{s: l \in L(s)} x_{s} \leq c_{l}, \quad \forall l \\
& \mathbf{x} \succeq 0
\end{array}
$$

where the variables are $\mathbf{x}$ and $\succeq$ means component-wise inequality between two vectors. Let $\mathrm{x}^{\text {opt }}$ be a globally optimal rate allocation that solves NUM.

The following basic assumption on utility functions are wellmotivated in many, although not all, applications and will still be maintained in this paper: utilities are functions of the allowed rates (rate-dependency), network utility is the sum of source utilities (additivity), and each source utility is an increasing (monotonicity) and local function of its own rate (locality). However, we will remove the standard yet often invalid assumption that utility functions are strictly concave and continuous.

Assuming that $U_{s}\left(x_{s}\right)$ becomes concave for large enough $x_{s}$ is reasonable, because the law of diminishing marginal utility eventually will be effective. However, $U_{s}$ may not be concave throughout its domain. Despite deficiency in the concavity assumption, almost all papers in the NUM literature for Internet rate allocation assume that utility functions are concave. Part of the reason is that the concavity assumption significantly simplifies the structure of the basic NUM problem (1) and leads to a distributed rate allocation algorithm as reviewed below.

Maximizing an additive concave function over linear constraints is a special case of convex optimization (minimizing a convex objective function over convex constraints [3]) called monotropic programming [16]. Thus, a local optimum is also a global optimum, and the duality gap ${ }^{1}$ is zero. Zero duality gap means that the minimized objective value of the Lagrange dual problem, which provides a decomposition structure, is equal to the maximized total utility in the primal problem (1).

The Lagrange dual problem of the basic NUM is readily derived. We first form the Lagrangian of (1)

$$
L(\mathbf{x}, \boldsymbol{\lambda})=\sum_{s} U_{s}\left(x_{s}\right)+\sum_{l} \lambda_{l}\left(c_{l}-\sum_{s: l \in L(s)} x_{s}\right)
$$

\footnotetext{
${ }^{1}$ Duality gap is the difference between the optimized dual objective value and
} the optimized primal objective value. where $\lambda_{l} \geq 0$ is the Lagrange multiplier (i.e., link price) associated with the linear flow constraint on link $l$. Additivity of total utility and linearity of flow constraints lead to a Lagrangian dual decomposition into individual source terms $L_{s}$

$$
\begin{aligned}
L(\mathbf{x}, \boldsymbol{\lambda}) & =\sum_{s}\left[U_{s}\left(x_{s}\right)-\left(\sum_{l \in L(s)} \lambda_{l}\right) x_{s}\right]+\sum_{l} c_{l} \lambda_{l} \\
& =\sum_{s} L_{s}\left(x_{s}, q_{s}\right)+\sum_{l} c_{l} \lambda_{l}
\end{aligned}
$$

where $q_{s}=\sum_{l \in L(s)} \lambda_{l}$ is the path price seen by source $s$. For each source $s, L_{s}\left(x_{s}, q_{s}\right)=U_{s}\left(x_{s}\right)-q_{s} x_{s}$ only depends on local rate $x_{s}$ and the path price $q_{s}$ (i.e., sum of $\lambda_{l}$ on links used by source $s$ ).

The Lagrange dual function $g(\boldsymbol{\lambda})$ is defined as the maximized $L(\mathbf{x}, \boldsymbol{\lambda})$ over $\mathbf{x}$ for a given $\lambda$. This 'net utility' maximization obviously can be conducted distributively by each source, as long as the aggregate link price $q_{s}$ is fed back to source $s$, where source $s$ maximizes $L_{s}\left(x_{s}, q_{s}\right)$ over $x_{s}$ for a given $q_{s}$

$$
x_{s}^{*}\left(q_{s}\right)=\arg \max \left[U_{s}\left(x_{s}\right)-q_{s} x_{s}\right], \quad \forall s .
$$

We can thus define a price-based rate allocation function for each source, also denoted as $x_{s}^{*}$, that maps $q_{s}$ into a maximizer of the partial Lagrangian $L_{s}$. Collecting such functions for all the sources, we write the Lagrangian maximizer vector as $\mathbf{x}^{*}(\boldsymbol{\lambda})$ where the argument is the vector of link prices.

The Lagrange dual problem of (1) is

$$
\begin{array}{ll}
\text { minimize } & g(\boldsymbol{\lambda})=L\left(\mathbf{x}^{*}(\boldsymbol{\lambda}), \boldsymbol{\lambda}\right) \\
\text { subject to } & \boldsymbol{\lambda} \succeq 0
\end{array}
$$

where the optimization variable is $\lambda$. It is well-known that, since $g(\boldsymbol{\lambda})$ is the point-wise supremum of a family of affine functions in $\boldsymbol{\lambda}$, it is convex and (3) is a convex minimization problem (even if the primal problem (1) is not a concave maximization problem).

An iterative subgradient method $^{2}$ can be used to update the dual variables $\boldsymbol{\lambda}$ to solve the dual problem (3)

$\lambda_{l}(t+1)=\left[\lambda_{l}(t)-\alpha(t)\left(c_{l}-\sum_{s: l \in L(s)} x_{s}^{*}\left(q_{s}(t)\right)\right)\right]^{+}, \quad \forall l$

where $(\delta g(\boldsymbol{\lambda}))_{l}=c_{l}-\sum_{s: l \in L(s)} x_{s}^{*}\left(q_{s}(t)\right)$ is the $l$ th component of a subgradient vector of $g(\boldsymbol{\lambda}), t$ is the iteration number, and $\alpha(t)>0$ are step sizes.

Certain choices of step sizes, such as $\alpha(t)=\beta / t$ for some constant $\beta>0$, guarantee that the sequence of dual variables $\lambda(t)$ converges to the dual optimal $\lambda^{\text {opt }}$ as $t \rightarrow \infty$. It can be shown that when the utility functions are concave, the primal variable $\mathbf{x}^{*}(\boldsymbol{\lambda}(t))$ also converges to the primal optimal variable $\mathbf{x}^{\mathrm{opt}}$. For a primal problem that is a convex optimization, the convergence is towards a global optimum. Rates $x_{s}$ are implicitly assumed to be upper bounded by finite numbers. Therefore, the global optimum can be attained since (1) is maximizing a continuous function over a compact set.

${ }^{2}$ A subgradient of a (possibly nondifferentiable) function $f: \mathbf{R}^{n} \rightarrow \mathbf{R}$ is any vector $\mathbf{g}$ such that, for any $\mathbf{x}$ in the domain of $f$, we have $f(\mathbf{y})-f(\mathbf{x}) \geq$ $\mathbf{g}^{T}(\mathbf{y}-\mathbf{x})$ for all $\mathbf{y}$ in the domain of $f$. 
In summary, we have the following well-known rate allocation algorithm for concave and continuous utility flows.

Algorithm 1-Price-Based Distributed Algorithm: The sequence of source and link algorithms (2), (4) forms a distributed subgradient algorithm that globally solves NUM (1) and the dual problem (3), and computes an optimal rate vector $\mathbf{x}^{\text {opt }}$ and optimal link price vector $\lambda^{\mathrm{opt}}$.

\section{Overview: Nonconcave or Discontinuous Utility}

Suppose we remove the critical assumption that $\left\{U_{s}\right\}$ are concave functions, and allow them to be any nonlinear functions. ${ }^{3}$ The resulting optimization problem becomes nonconvex and significantly harder to analyze and solve, even by centralized computational methods. In particular, a local optimum may not be a global optimum and the duality gap can be strictly positive. The standard distributive algorithms that solve the dual problem may produce infeasible or suboptimal rate allocation. Global maximization of nonconcave functions is an intrinsically difficult problem of nonconvex optimization. Indeed, over the last two decades, it has been widely recognized that "in fact the great watershed in optimization isn't between linearity and nonlinearity, but convexity and nonconvexity" (Rockafellar, [17]).

When some source utilities are nonconcave, it appears that Algorithm 1 will not converge or will converge to only a locally optimal rate allocation, because it is based on solving the dual problem and yet the duality gap can be strictly positive. However, we show in Section II that, even when all source utilities are nonconcave functions, Algorithm 1 may still converge to a globally optimal rate allocation and duality gap may still be zero. We then prove that continuity of price-based rate allocation $\mathrm{x}^{*}(\boldsymbol{\lambda})$ at all the optimal prices $\boldsymbol{\lambda}^{\mathrm{opt}}$ is a sufficient condition for global convergence, and its continuity at at least one of the optimal prices is a necessary condition.

The sufficient condition leads to the idea of provisioning link capacities so as to achieve convergence of Algorithm 1 to the global optimum. Intuitively, if link capacities can be provisioned such that the optimal price $q_{s}^{\text {opt }}$ for a nonconcave utility source $s$ is steered away from the points where $\mathbf{x}_{s}^{*}$ is discontinuous, then Algorithm 1 is guaranteed to converge to the globally optimal rate allocation. This intuition is made rigorous in Section III, where we derive the set of link capacity vector $\mathbf{c}$ that ensures global convergence of Algorithm 1 and formulate the problem of computing the appropriate amount of link capacity provisioning. This section reveals the connection between link capacity configuration and the effects of source elasticity, and the results can be used to conduct offline design of network capacity, striking a tradeoff between provisioning link capacities and accommodating inelastic flows.

Then in Section IV, we turn to the case of discontinuous utility function by considering a network with real-time flows sharing link capacities with elastic flows. There are three key questions addressed in this case: when will the optimal solution be to admit all the real-time flows (with type (b) inelastic utility in Fig. 1)? If admission control is needed for real-time flows,

\footnotetext{
${ }^{3}$ Sometimes a nonconcave function can be easily turned into a concave one by a simple transformation, for example in the case of the "pseudo-nonconvexity" in the power control problems in [4], [7]. Here we are concerned with nonconcave functions that cannot be readily turned into concave ones.
}

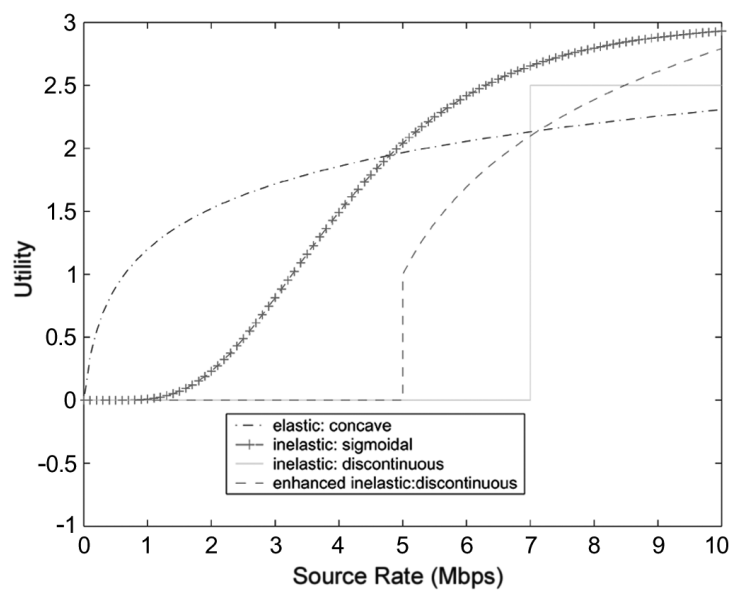

Fig. 1. Some examples of utility functions $U_{s}\left(x_{s}\right)$ : it can be concave, sigmoidal, discontinuous, or any general nonconcave function. Elastic flows have concave utilities, inelastic flows have (a) nonconcave utilities or (b) discontinuous utilities that stays at a constant level after a threshold, and enhanced real-time flows have (c) discontinuous utilities that is concave after a threshold.

how should it be carried out at the network edge? If all real-time flows can be admitted and they follow type (c) inelastic utility in Fig. 1, how to protect the elastic flows so that they are guaranteed a share of the link capacities? We answer the first question by applying the idea of capacity provisioning, answer the second question by proposing a price-based, two-phase admission control heuristic, and answer the third question by proposing an optimal algorithm that guarantees a minimum bandwidth share to elastic flows.

Results in this work further advances the method of distributed rate allocation in practically important cases where some flows are inelastic. Numerical examples are used to illustrate the key results throughout the paper. A summary of the recent approaches to distributed rate allocation with nonconcave and discontinuous utilities is presented in Table I.

\section{NonConCAVE Utility Flows: Optimality CONDITIONS}

With nonconcave utility functions, Algorithm 1 may fail to converge to the primal optimal solution $\mathbf{x}^{\text {opt }}$. One reason is that solving the dual problem (3) is no longer equivalent to solving the primal problem (1). In the case of allocating rates by solving NUM, it is the primal problem that we care about. Lee, Mazumdar, and Shroff [11] show that, in the case of sigmoidal utilities, Algorithm 1 may cause link congestion and produce suboptimal rate allocation. A 'self-regulating' heuristics is proposed, and is shown to avoid link congestion caused by sigmoidal utilities, but may not attain the optimal rate allocation $\mathrm{x}^{\mathrm{opt}}$ except in the asymptotic case when the proportion of self-regulated sources that stop transmitting data vanishes.

In this section, we study the general case where $\left\{U_{s}\right\}$ are nonconcave functions. The goal of our study is to investigate sufficient and necessary conditions ${ }^{4}$ under which Algorithm 1 still converges to the globally optimal rate allocation, i.e., the primal optimizer $\mathbf{x}^{\text {opt }}$.

${ }^{4}$ When these conditions do not hold, a centralized computational method based on the sum-of-squares approach has recently been studied [8] and is empirically found to compute the globally optimal rate allocation efficiently. However, this approach is not amenable to distributed algorithms. 
TABLE I

Summary of DifFERENT APPROACHES OF Distributed RATE AlLOCATION FOR INELASTIC Flows

\begin{tabular}{|l|l|l|}
\hline & Nonconcave Utility & Discontinuous Utility \\
\hline Sufficient condition for Algorithm 1's convergence and optimality & Section II & Section II \\
\hline Capacity provisioning for Algorithm 1's convergence and optimality & Section III (sigmoidal utility only) & Section IV-A \\
\hline Heuristics for suboptimal but distributed solution & Ref. [11] (sigmoidal utility only) & Section IV-B \\
\hline Sum-of-squares method for optimal but centralized solution & Ref. [8] (polynomial utility only) & \\
\hline
\end{tabular}

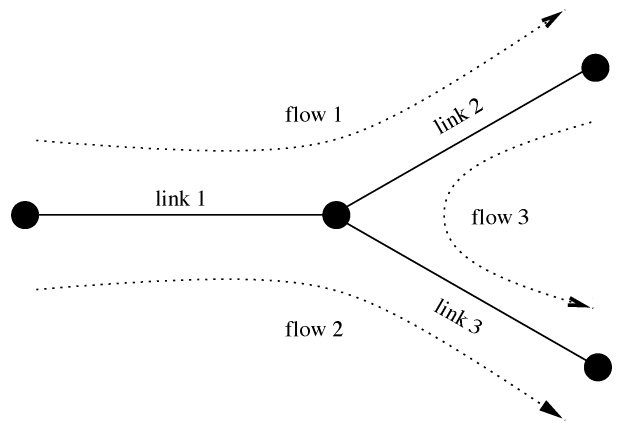

Fig. 2. Network topology for Example 1 and Example 4.

\section{A. Does Algorithm 1 Work for Nonconcave Sources?}

The following example illustrates that Algorithm 1 can converge even when the utility functions are nonconcave.

Example 1: There are three flows over three links as shown in Fig. 2. The small number of flows and links allow exhaustive search to compute the global optimum and check against distributed algorithm's solution. All three utility functions are nonconcave: $U_{s}\left(x_{s}\right)=\left(1-2 Q\left(\sqrt{2 x_{s}}\right)\right)^{\beta_{s}}$ where $Q$ is the complementary cumulative distribution of standard Gaussian variable and $\beta_{s}$ are positive parameters. The link capacity vector is varied within the set $\mathcal{C}=\{\mathbf{c}(\theta)=\theta[5,10,6]+(1-\theta)[8,6,7], \theta \in$ $[0,1]\}$. Each capacity vector gives one realization of a nonconcave NUM. Algorithm 1 is executed for this problem and the resulting rate allocation is indeed found to be globally optimal for all $\mathbf{c} \in \mathcal{C}$. As shown in Fig. 3 for some of the choices of c, the maximized network utility through Algorithm 1 matches precisely with that from exhaustive search.

A natural question thus arises: under what conditions will Algorithm 1 converge to the globally optimal rate allocation for nonconcave NUM? In short, when will Algorithm 1 'work' for inelastic flow rate allocation? One necessary condition that immediately manifests itself is the zero duality gap condition.

\section{B. Necessary Condition: Zero Duality Gap}

Let $U^{*}$ be the globally optimal primal objective value and $\mathrm{x}^{\mathrm{opt}}$ a maximizer, i.e., $U^{*}=\sum_{s} U_{s}\left(x_{s}^{\mathrm{opt}}\right)$, and $D^{*}$ be the globally optimal dual objective value and $\lambda^{\text {opt }}$ a minimizer, i.e., $D^{*}=g\left(\boldsymbol{\lambda}^{\text {opt }}\right)$. It is well-known that, irrespective of the concavity of the utilities, $D^{*} \geq U^{*}$ [3]. The duality gap for (1) is defined as $\eta=D^{*}-U^{*}$ and we have $\eta \geq 0$ in general. The zero duality gap condition means that $\eta=0$. It is easy to see that zero duality gap is a necessary condition for Algorithm 1 to converge to primal optimal. Algorithm 1 solves the basic NUM through its Lagrange dual problem (3). The primal variable that the algorithm converges to can be a solution of the original NUM problem only if the duality gap is zero.

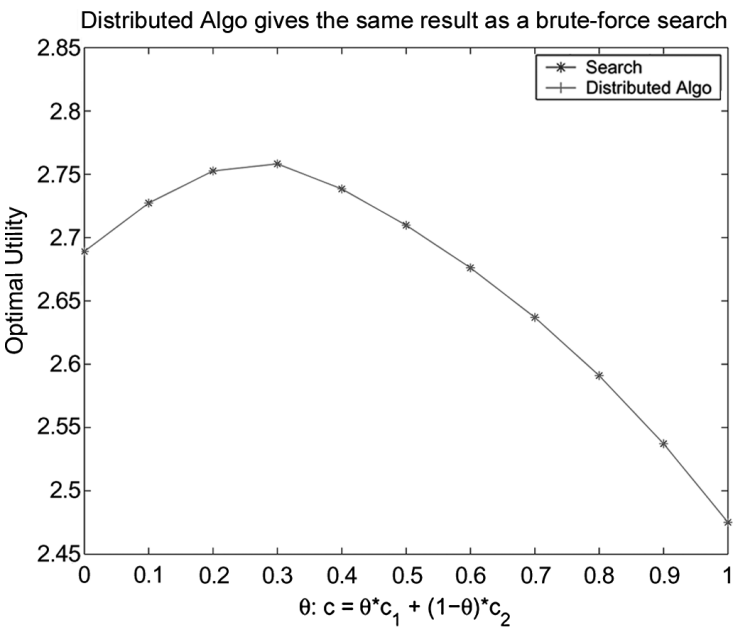

Fig. 3. (Example 1) Distributed subgradient algorithm may still converge to globally optimal rate allocation despite nonconcavity of $U_{s}\left(x_{s}\right)$. A cross-sectional sample is shown in the graph where Algorithm 1's converged results coincide with the globally optimal rate allocation computed through exhaustive search.

While concavity of all utility functions $\left\{U_{s}\left(x_{s}\right)\right\}$ is a sufficient condition to guarantee zero duality gap in (1), it is not a necessary condition. Duality gap can be zero even for nonconvex optimization problems. However, proving zero duality gap in these cases can be much more difficult and requires arguments beyond the standard ones in convex optimization [3]. In this subsection, we demonstrate a more general sufficient condition for duality gap to be zero, which includes the concavity condition of utility functions as a special case, but shows that duality gap can be zero even for nonconcave utilities.

Consider the optimized total utility $U^{*}=\sum_{s} U_{s}\left(x_{s}^{\text {opt }}\right)$ as a function of link capacities: $U^{*}(\mathbf{c})$. This is a completely different function from the utility functions $\left\{U_{s}\left(x_{s}\right)\right\}$, which are functions of source rates. As long as $U^{*}(\mathbf{c})$ is a concave function, the duality gap of (1) is zero under mild technical conditions. There is a subtle but significant difference between concavity of $U_{s}\left(x_{s}\right)$, i.e., concavity of each user's utility as a function of rate, and the more general concavity of $U^{*}(\mathbf{c})$, i.e., concavity of the optimized total utility as a function of link capacities. The former implies the latter, but not vice versa. In Example 1, it can be verified that $U^{*}(\mathbf{c})$ is concave for $\mathbf{c} \in \mathcal{C}$ even though the individual utility functions are not all concave.

The condition is stated formally as follows. For each $\mathbf{c}$ in a set $\mathcal{C}$ of possible link capacity vectors, there is a set of feasible $\mathbf{x}$ for (1), which defines a set of achievable values of total utility $U(\mathbf{c})$. The following lemma follows directly from the 'min common max crossing duality' principle in nonlinear optimization theory [2]: 


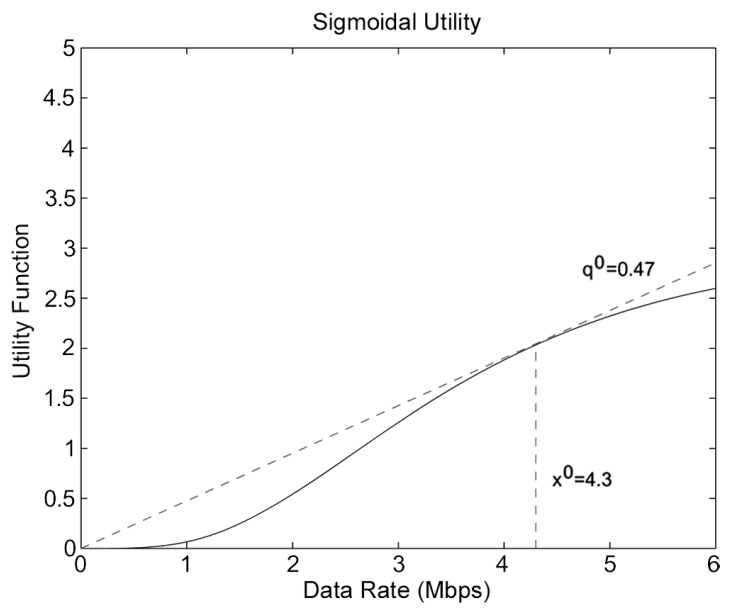

Fig. 4. Sigmoidal utility function and illustration of $x^{0}$ and $q^{0}$.

Lemma 1: If $U^{*}(\mathbf{c})$ is a concave function, duality gap $\eta=$ $D^{*}-U^{*}$ for $(1)$ is zero.

While zero duality gap is a necessary condition for convergence of Algorithm 1 to the global optimum, additional conditions are required to ensure that the price-based rate allocation resulting from the algorithm also converges to the optimal solution of the original problem, as illustrated through the following example.

Example 2: Consider a simple network: two flows sharing a link with capacity $c$. One flow is elastic data traffic, with logarithmic (concave) utility function, and the other is inelastic traffic, with sigmoidal (nonconcave) utility function shown in Fig. 4. We will demonstrate that, if the capacity $c$ has a particular value, then zero-duality gap holds for the problem and yet Algorithm 1 does not provide the optimal rate allocation.

For any given sigmoidal curve $U_{2}\left(x_{2}\right)$, construct a straight line from the origin to be tangent to the sigmoidal curve. Denote the $\mathrm{x}$-coordinate of the intersection of the tangent with the curve by $x_{2}^{0}$ and the slope of the tangent by $q^{0}$. It is easy to verify that $\left\{0, x_{2}^{0}\right\}=x_{2}^{*}\left(q^{0}\right)$, i.e., both 0 and $x_{2}^{0}$ maximize $U_{2}\left(x_{2}\right)-q^{0} x_{2}$. Consider $c=x_{1}^{*}\left(q^{0}\right)+x_{2}^{0}$. In this case, the dual optimal price $q^{\text {opt }}=q^{0}$ because it can verified that any subgradient to the dual function is zero at $q^{0}$ (if not, a nonzero subgradient can be verified to change sign when evaluated on either side of $q^{0}$ in an $\epsilon$ neighborhood of $q^{0}$, i.e., $\left.\left\{q^{0}-\epsilon, q^{0}+\epsilon\right\}\right)$.

At the dual optimal $q^{\text {opt }}=q^{0}$, we have both $\left\{x_{1}^{*}\left(q^{0}\right), 0\right\}$ and $\left\{x_{1}^{*}\left(q^{0}\right), x_{2}^{0}\right\}$ as the price-based rate allocation. We have $D^{*}=g\left(q^{0}\right)=U_{1}\left(x_{1}^{*}\left(q^{0}\right)\right)+U_{2}\left(x_{2}^{0}\right)$, and $U^{*} \geq U_{1}\left(x_{1}^{*}\left(q^{0}\right)\right)+U_{2}\left(x_{2}^{0}\right)$ by definition of primal optimality and feasibility of $\left\{x_{1}^{*}\left(q^{0}\right), x_{2}^{0}\right\}$. So we have $U^{*} \geq D^{*}$. But we also have $D^{*} \geq U^{*}$ by weak duality. Therefore, $D^{*}=U^{*}$, i.e., the duality gap is zero at both the price-based rate allocations. However, only one of the price-based rate allocation is also a primal optimal, the pair $\left\{x_{1}^{*}\left(q^{0}\right), x_{2}^{0}\right\}$. The other pair $\left\{x_{1}^{*}\left(q^{0}\right), 0\right\}$ is not a primal optimal but still satisfies the zero-duality gap condition.

Note that this example is a constructed scenario where the capacity was just enough to achieve zero-duality gap but still not enough to result in convergence of the price-based rate allocation to the primal optimal. If the capacity is slightly larger than this minimum value, then the price-based rate allocation for the sigmoidal function becomes continuous and Algorithm 1 converges to the primal optimal.

\section{Sufficient Condition: Continuity of Price-Based Source Rates}

In this subsection, we provide a sufficient condition under which Algorithm 1 converges. This sufficient condition subsumes the well-known sufficient condition of concavity of the utility functions for convergence. The condition is stated formally in the following theorem.

Theorem 1: Continuity of price-based rate allocation $\mathrm{x}^{*}(\boldsymbol{\lambda})$ at all optimal prices $\lambda^{\text {opt }}$ implies that the price-based rate allocation $\mathrm{x}^{*}(\boldsymbol{\lambda})$ obtained through Algorithm 1 converges to a globally optimal rate allocation $\mathrm{x}^{\mathrm{opt}}$.

Proof: First we claim that continuity of price-based rate allocation $\mathbf{x}^{*}(\boldsymbol{\lambda})$ at the optimal price $\boldsymbol{\lambda}^{\mathrm{opt}}$ implies that $\mathbf{x}^{*}\left(\boldsymbol{\lambda}^{\mathrm{opt}}\right)$ and $\lambda^{\text {opt }}$ satisfy complementary slackness and that $\mathbf{x}^{*}\left(\boldsymbol{\lambda}^{\text {opt }}\right)$ is primal feasible. The complementary slackness condition between the optimal congestion price and price-based rate allocation is given by

$$
\lambda_{l}^{\mathrm{opt}}\left(\sum_{s: l \in L(s)} x_{s}^{*}\left(q_{s}^{\mathrm{opt}}\right)-c_{l}\right)=0, \quad \forall l .
$$

To see this, for any given $l$, consider first the case where $\lambda_{l}^{\text {opt }}>$ 0 . Let $\epsilon>0$ be sufficiently small such that we can define price vectors $\lambda^{\text {min }}$ where $\left\{\lambda_{i}^{\min }=\lambda_{i}^{\text {opt }}, i \neq l ; \lambda_{l}^{\min }=\lambda_{l}^{\text {opt }}-\epsilon>0\right\}$ and $\lambda^{\max }$ where $\left\{\lambda_{i}^{\max }=\lambda_{i}^{\text {opt }}, i \neq l ; \lambda_{l}^{\max }=\lambda_{l}^{\text {opt }}+\epsilon>0\right\}$. By the definition of subgradient, we have

$$
\begin{aligned}
g\left(\boldsymbol{\lambda}^{\mathrm{opt}}\right) & \geq g\left(\boldsymbol{\lambda}^{\mathrm{min}}\right)+\left(\boldsymbol{\lambda}^{\mathrm{opt}}-\boldsymbol{\lambda}^{\mathrm{min}}\right)^{T} \delta g\left(\boldsymbol{\lambda}^{\mathrm{min}}\right) \\
& =g\left(\boldsymbol{\lambda}^{\mathrm{min}}\right)+\epsilon\left(c_{l}-\sum_{s: l \in L(s)} x_{s}^{*}\left(\boldsymbol{\lambda}^{\mathrm{min}}\right)\right)
\end{aligned}
$$

Since $g\left(\boldsymbol{\lambda}^{\mathrm{opt}}\right) \leq g\left(\boldsymbol{\lambda}^{\mathrm{min}}\right)$, we have that the $l$ th component of the subgradient evaluated at $\lambda^{\text {min }}$ is nonpositive, resulting in

$$
\sum_{s: l \in L(s)} x_{s}^{*}\left(\lambda^{\min }\right) \geq c_{l} .
$$

Similarly, the $l$ th component of the subgradient evaluated at $\lambda^{\max }$ is nonnegative, resulting in

$$
\sum_{s: l \in L(s)} x_{s}^{*}\left(\lambda^{\max }\right) \leq c_{l} .
$$

By continuity of $\mathbf{x}^{*}(\boldsymbol{\lambda})$ at $\boldsymbol{\lambda}^{\mathrm{opt}}, \sum_{s: l \in L(s)} x_{s}^{*}\left(q_{s}^{\mathrm{opt}}\right)=c_{l}$, which implies both complementary slackness and primal feasibility.

Next, consider the case $\lambda_{l}^{\text {opt }}=0$. While complementary slackness condition is obvious, we need to show that the pricebased rate allocation is primal feasible. Let $\epsilon>0$, and $\lambda_{l}^{\max }$ and $\lambda^{\max }$ be as defined before. The $l$ th component of the subgradient at $\lambda^{\max }$ is nonnegative

$$
\sum_{s: l \in L(s)} x_{s}^{*}\left(\lambda^{\max }\right) \leq c_{l} .
$$

By continuity of $\mathbf{x}^{*}(\lambda)$ at $\lambda^{\mathrm{opt}}, \sum_{s: l \in L(s)} x_{s}^{*}\left(q_{s}^{\mathrm{opt}}\right) \leq c_{l}$ in this case, proving primal feasibility. 
We now show that $D^{*} \leq U^{*}$

$$
\begin{aligned}
D^{*} & \stackrel{(a)}{=} g\left(\boldsymbol{\lambda}^{\mathrm{opt}}\right) \\
& \stackrel{(b)}{=} \max _{\mathbf{x}} L\left(\mathbf{x}^{*}\left(\boldsymbol{\lambda}^{\mathrm{opt}}\right), \boldsymbol{\lambda}^{\mathrm{opt}}\right) \\
& \stackrel{(c)}{=} \sum_{s} U_{s}\left(x_{s}^{*}\left(q_{s}^{\mathrm{opt}}\right)\right)+\sum_{l} \lambda_{l}^{\mathrm{opt}}\left(c_{l}-\sum_{s: l \in L(s)} x_{s}^{*}\left(q_{s}^{\mathrm{opt}}\right)\right) \\
& \stackrel{(d)}{=} \sum_{s} U_{s}\left(x_{s}^{*}\left(q_{s}^{\mathrm{opt}}\right)\right) \\
& \stackrel{(e)}{\leq} U^{*}
\end{aligned}
$$

where (a) follows from the definition of dual optimal value, (b) from the definition of Lagrange dual function, (c) from the definition of Lagrangian, (d) from complementary slackness, and (e) from the definition of primal optimal value. But then, by weak duality, $D^{*} \geq U^{*}$. Thus, $D^{*}=U^{*}$, i.e., inequality (e) must be an equality. Therefore, price-based rate allocation $\mathbf{x}^{*}\left(\boldsymbol{\lambda}^{\mathrm{opt}}\right)$ is a feasible rate vector that attains the globally optimal network utility, i.e., Algorithm 1 converges to the globally optimal rate allocation.

A consequence of Theorem 1 is that the continuity property implies zero duality gap, and further results in the convergence of Algorithm 1 to the primal optimal. We revisit the previous example to illustrate this property.

Example 3: Consider the simple network in Example 2 with two flows sharing a link with capacity $c$. One flow is elastic data traffic, with logarithmic utility function, and the other is inelastic traffic, with sigmoidal utility function shown in Fig. 4. Let $q^{0}$ and $x_{2}^{0}$ be as defined in Example 2. We vary the link capacity $c$ and observe the behavior of the convergence of Algorithm 1 to the global optimum. We observe that for $c<x_{1}^{*}\left(q^{0}\right)+x_{2}^{0}$, we have neither zero duality gap nor convergence of Algorithm 1 to primal optimum. For $c=x_{1}^{*}\left(q^{0}\right)+x_{2}^{0}$, we have zero duality gap but Algorithm 1 does not converge. But for $c>x_{1}^{*}\left(q^{0}\right)+x_{2}^{0}$, continuity property in Theorem 1 holds, duality gap is zero, and Algorithm 1 converges.

As shown in Fig. 5, as link capacity becomes larger than a threshold, i.e., $c>5.38$, the optimal dual variable $q^{\text {opt }}<q^{0}$, thus ensuring continuity of price-based rate allocation, and Algorithm 1 converges to the primal optimal. The convergence is shown by a time plot in Figs. 6 and 7 where we see that Algorithm 1 converges only if the capacity is larger than the minimum required $x_{1}^{*}\left(q^{0}\right)+x_{2}^{0}$. In fact, for $c \leq x_{1}^{*}\left(q^{0}\right)+x_{2}^{0}$, the algorithm is deceptively convergent with occasionally transitions to zero source rate for the inelastic sigmoidal source. The closer the link capacity is to the minimum required for convergence, the less frequent the transitions to zero rate.

If $\mathbf{x}^{*}(\boldsymbol{\lambda})$ is discontinuous at all optimal prices $\boldsymbol{\lambda}^{\text {opt }}$, Algorithm 1 certainly cannot converge. This observation readily leads to the following necessary condition:

Theorem 2: Continuity of $\mathrm{x}^{*}(\boldsymbol{\lambda})$ at at least one of the optimal prices $\boldsymbol{\lambda}^{\text {opt }}$ is a necessary condition for Algorithm 1 to converge to a globally optimal rate allocation.

If the dual objective function $g(\boldsymbol{\lambda})$ is strictly convex (note that it is always convex), even if it is nondifferentiable, there is a unique optimal price. Therefore, we have the following corollary.

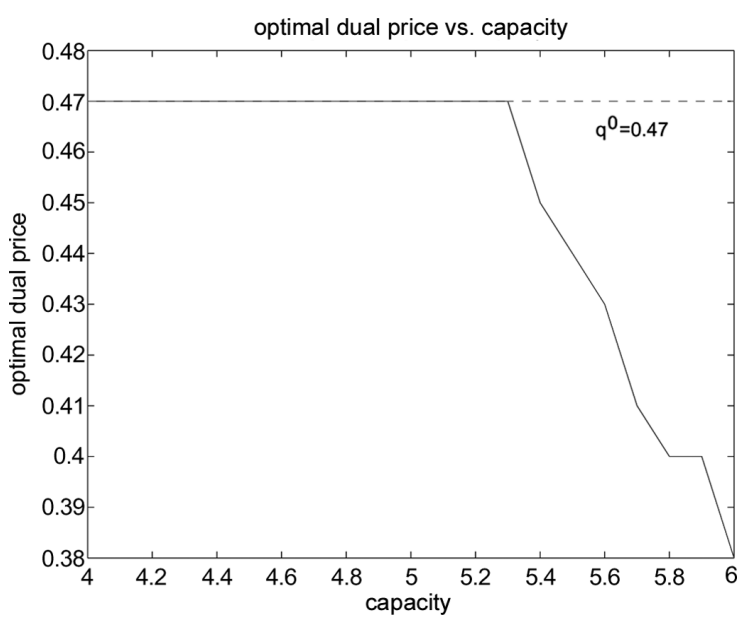

Fig. 5. (Example 3) The dual optimal $\lambda^{\mathrm{opt}}$ as a function of the link capacity $c$.

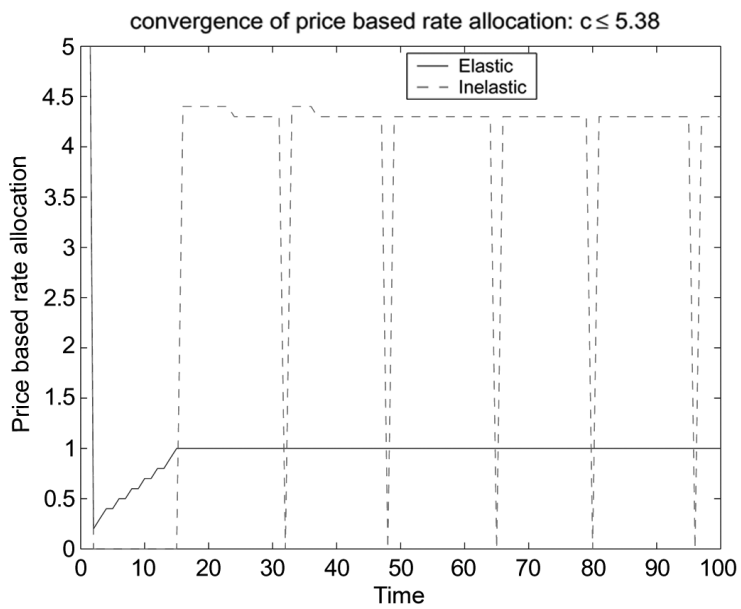

Fig. 6. (Example 3) Price-based rate allocation when link capacity $c \leq 5.38$ Rate allocation for inelastic source does not converge.

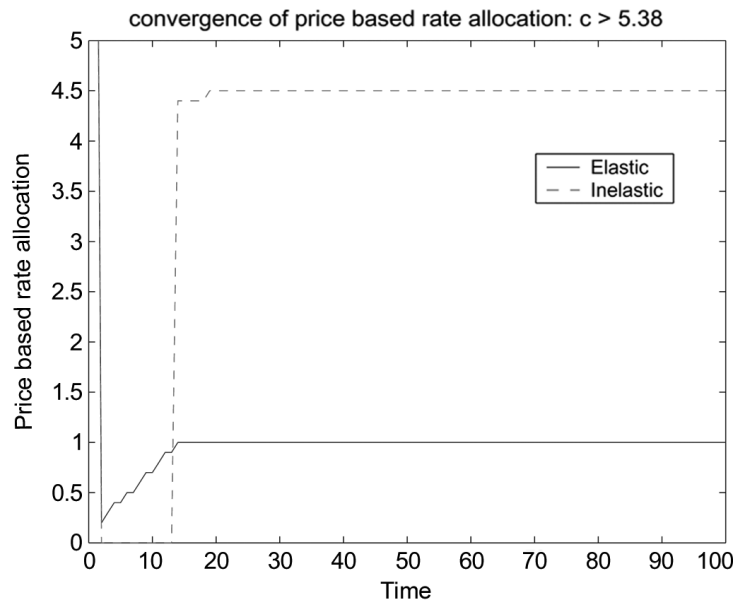

Fig. 7. (Example 3) Price-based rate allocation when link capacity $c>5.38$. Rate allocation for both sources converge.

Corollary 1: If $g(\boldsymbol{\lambda})$ is strictly convex, continuity of $\mathbf{x}^{*}(\boldsymbol{\lambda})$ at $\lambda^{\text {opt }}$ is a necessary and sufficient condition for Algorithm 1 to converge to the globally optimal rate allocation. 


\section{Nonconcave Utility Flows: CAPACITY PROVISIONING FOR CONVERGENCE OF ALGORITHM 1}

\section{A. Motivation}

As shown in Section II-C, it is possible to achieve convergence of Algorithm 1 to the optimal rate allocation even with nonconcave utility functions, as long as certain path prices are avoided. This section points out the relationship between link capacity provisioning and convergence of Algorithm 1, and quantifies the tradeoff between the cost of capacity provisioning and the need for the standard price-based rate allocation algorithm (e.g., TCP congestion control variants) to converge. Results in this section can thus be used for both convergence analysis and offline link capacity design.

We focus on the case of sigmoidal utilities in this section. An intuitive guess is that Algorithm 1 would converge if link capacities can guarantee that the optimal rate for a sigmoidal utility source lies in the concave part of the sigmoidal curve. It turns out this intuition is imprecise. We will develop the correct intuition in this subsection, followed by quantitative characterization and numerical examples in the next three subsections.

Let $U_{s}\left(x_{s}\right)$ be a sigmoidal function as shown in Fig. 4. It is strictly increasing, has one inflection point $x_{s}^{i}$, and $\left(d^{2} U_{s}\left(x_{s}\right) / d x_{s}\right)>0$, for $x_{s}<x_{s}^{i}$ and $\left(d^{2} U_{s}\left(x_{s}\right) / d x_{s}\right)<0$, for $x_{s}>x_{s}^{i}$. Let $q_{s}^{0}$ be the slope of the tangent from the origin to the sigmoidal curve $U_{s}\left(x_{s}\right)$. Let $x_{s}^{0}$ be the source rate at which the tangent intersects the sigmoidal curve $U_{s}\left(x_{s}\right)$. Theorem 1 shows that continuity of $\mathbf{x}^{*}(\boldsymbol{\lambda})$ at the dual optimal $\lambda^{\text {opt }}$ implies convergence of Algorithm 1 and primal optimality of $\mathbf{x}^{*}\left(\boldsymbol{\lambda}^{\mathrm{opt}}\right)$. We note that for the sigmoidal function $U_{s}\left(x_{s}\right)$, $x_{s}^{*}(\boldsymbol{\lambda})$ is discontinuous when $q_{s}=q_{s}^{0}$. For $q_{s}>q_{s}^{0}$, we have $x_{s}^{*}(\boldsymbol{\lambda})=0$ and the source gets 0 rate. On the other hand, for $q_{s}<q_{s}^{0}$, we have $x_{s}^{*}(\boldsymbol{\lambda})>x_{s}^{0}$ and the source gets positive rate and utility. If the optimal price $\lambda^{\text {opt }}$ is such that $q_{s}^{\text {opt }}<q_{s}^{0}$ for every sigmoidal utility source, then $x_{s}^{*}\left(\boldsymbol{\lambda}^{\mathrm{opt}}\right)$ is continuous at the optimal price, and the sufficient condition is met. Algorithm 1 can then be applied to this nonconvex optimization problem to obtain the global optimal.

The optimal price on a link depends on the link capacity and the load offered by all the sources using the link. In general, the link capacities can be increased to bring down the optimal prices to a desired value that ensures the convergence of Algorithm 1. Naturally we would like to use the minimal set of capacities that would ensure continuity of the price-based rate allocation. We quantify this idea in the rest of this section.

\section{B. Capacity Provisioning for Convergence}

Consider a network with $L$ links and $S$ sources as in Section I-B. Let $S_{n}$ of the sources have a nonconcave sigmoidal utility, and, without loss of generality, let these be the first $S_{n}$ of the $S$ sources. The other $S_{c}=S-S_{n}$ sources have concave utility functions. Without loss of generality, we assume that all of the $L$ links considered serve some nonconcave sigmoidal source, because otherwise the links that do not serve any sigmoidal source can be ignored (increasing the capacity on those links does not contribute towards reducing the prices seen by the sigmoidal sources).

Let the routing matrix corresponding to the $S_{n}$ sigmoidal sources be a $L \times S_{n}$ matrix $\mathbf{R}_{n}$ (where the $(l, s)$ entry of the routing matrix is 1 if $l \in L(s)$ and 0 otherwise) and the routing matrix of the other $S_{c}$ sources be $\mathbf{R}_{c}$, so that $\mathbf{R}=\left[\mathbf{R}_{n} \mathbf{R}_{c}\right]$ is the routing matrix of the entire network. For sigmoidal utility sources, let $\mathbf{x}_{n}^{0}$ and $\mathbf{q}_{n}^{0}$ be the vector of source rates and slopes, respectively, at the point where the tangent from the origin intersects the sigmoidal curves.

Theorem 3: Consider the NUM problem with source utilities a mix of strictly concave and sigmoidal functions as modeled in this section. Algorithm 1 applied to this NUM problem converges to the primal optimal rate allocation, and sources with sigmoidal utilities obtain nonzero source rates, if there is a $\mathbf{p} \succeq 0$ satisfying the following inequality 5

$$
\mathbf{q}_{n}^{0} \succ \mathbf{R}_{n}^{T} \mathbf{p}
$$

and the link capacities $\mathbf{c}=\mathbf{c}(\mathbf{p})$ where

$$
\mathbf{c}(\mathbf{p})=\mathbf{R}_{n} \mathbf{x}_{n}^{*}(\mathbf{p})+\mathbf{R}_{c} \mathbf{x}_{c}^{*}(\mathbf{p}) .
$$

Here, $\mathbf{x}_{n}^{*}(\mathbf{p})$ and $\mathbf{x}_{c}^{*}(\mathbf{p})$ are the price-based rate allocation at price $\mathbf{p}$ obtained by solving (2) for sources with nonconcave and concave utilities, respectively.

Proof: First recall that the subgradient of the Lagrangian dual function $g(\boldsymbol{\lambda})$ is given by

$$
\delta g(\boldsymbol{\lambda})=\mathbf{c}-\mathbf{R x}^{*}(\boldsymbol{\lambda})
$$

If $\mathbf{c}=\mathbf{c}(\mathbf{p})$, then the subgradient at price $\mathbf{p}$ vanishes, i.e., $\delta g(\mathbf{p})=0$. The solution to the dual problem (3), $\lambda^{\mathrm{opt}}$, is unique [Proposition 1 in [11]] under the modeling assumption in this section. This implies that $\lambda^{\mathrm{opt}}=\mathbf{p}$. For the nonconcave sources, we have $q_{s}^{\text {opt }}=\left(\mathbf{R}_{n}^{T} \mathbf{p}\right)_{s}$ and $q_{s}^{0} \geq\left(\mathbf{R}_{n}^{T} \mathbf{p}\right)_{s}$. So we have $q_{s}^{\text {opt }}<q_{s}^{0}$. This implies that the sigmoidal sources have an optimal pricing that is less than the critical pricing $q_{s}^{0}$, hence the price-based rate allocation $x_{s}^{*}\left(q_{s}^{\mathrm{opt}}\right)$ is continuous, implying that Algorithm 1 converges to primal optimal. Furthermore, $x_{s}^{*}\left(q_{s}^{\mathrm{opt}}\right)$ is strictly positive, i.e., rate allocation to each source is nonzero. ${ }^{6}$

Remark 1: Note that the capacities $\mathbf{c}(\mathbf{p})$ are larger for smaller prices $\mathbf{p}$. This result highlights the following interesting relationship between provisioning link capacity and ensuring the applicability of standard price-based rate allocation: If link capacities are sufficiently large, Algorithm 1 can still converge to the globally optimal rate allocation despite nonconcavity of source utilities. Furthermore, since $\mathrm{x}^{*}$ is nonincreasing in $\mathbf{p}$, we conclude that the more inelastic the flows are (i.e., smaller $\mathbf{q}_{n}^{0}$ ), the larger the link capacities needed for convergence. Source elasticity can be traded-off with link capacity.

Remark 2: Given a price vector $\mathbf{p}$ that satisfies (5), the capacities $\mathbf{c}(\tilde{\mathbf{p}})$ that correspond to the set of prices $\tilde{\mathbf{p}} \preceq \mathbf{p}$ does not span the entire set of capacities $\mathbf{c} \succeq \mathbf{c}(\mathbf{p})$. We define $\mathcal{C}_{s}$ as the set of capacities that satisfy the conditions of Theorem 3. So $\mathcal{C}_{s}=\{\mathbf{c}: \mathbf{c}=\mathbf{c}(\mathbf{p})$ for some $\mathbf{p}$ that satisfies (5) $\}$.

${ }^{5}$ Note that $\mathbf{p}$ is a generic representation of the dual price vector based on which the capacity requirements have been specified. We reserve the notation $\boldsymbol{\lambda}$ for the price vector associated with Algorithm 1.

${ }^{6}$ An alternative proof to the theorem can be obtained by noting that the vanishing subgradient implies complementary slackness and hence $\mathbf{x}^{*}(\mathbf{p})$ is indeed the primal optimum. 


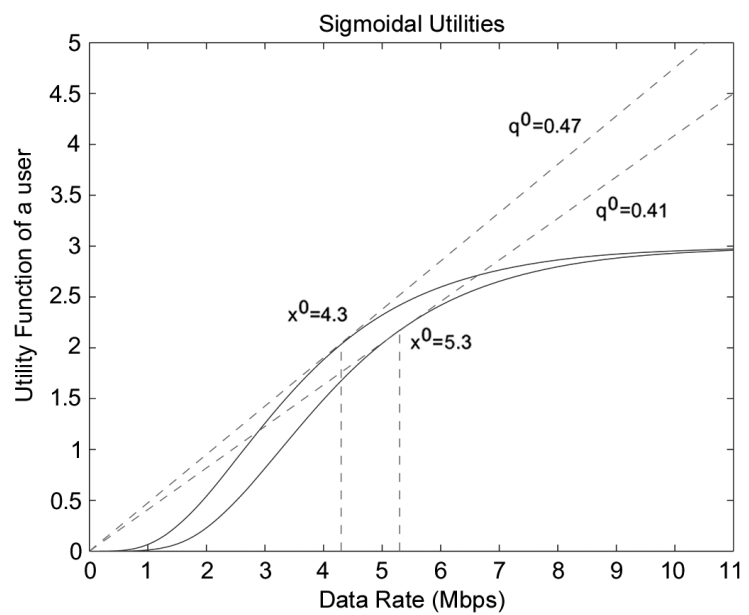

Fig. 8. (Example 4) Sigmoidal utilities for the two sources.

Remark 3: Convergence to primal optimal can be achieved even at capacities lower than that specified in Theorem 3. If the capacity vector satisfies the following condition:

$$
\begin{aligned}
\mathbf{c} & \prec \mathbf{R}_{c} \mathbf{x}_{c}^{*}(\mathbf{p}) \\
\mathbf{q}_{n}^{0} & \preceq \mathbf{R}_{n}^{T} \mathbf{p}
\end{aligned}
$$

then Algorithm 1 also converges to the primal optimal. However, the primal optimal solution in this case allocates zero rate to all sigmoidal utility sources and this situation is not of much practical interest. Let $\mathcal{C}_{n}$ be the set of capacities that satisfy (7).

Therefore, $\mathcal{C}_{s}$ is the set of capacities that achieves convergence and allocates strictly positive rates to sigmoidal sources, while $\mathcal{C}_{n}$ is the set of capacities that achieves convergence but allocates zero source rates to sigmoidal sources. For capacities that belong to neither $\mathcal{C}_{s}$ nor $\mathcal{C}_{n}$, convergence is unclear and remains to be characterized.

Based on Theorem 3, we propose the following algorithm for capacity provisioning in a network.

Algorithm 2-Capacity Provisioning: For a network with any combination of elastic and inelastic flows:

- Pick a price vector $\mathbf{p}$ that satisfies (5).

- Calculate the capacity vector as $\mathbf{c}=\mathbf{c}(\mathbf{p})$ as in (6).

Example 4: To confirm the predictions by Theorem 3, consider the network topology shown in Fig. 2. The network consists of three links shared by three flows. The first two flows have sigmoidal utilities and the third one has a concave utility. As shown in Fig. 8, the two sigmoidal utilities have $q_{1}^{0}=0.41$ and $q_{2}^{0}=0.47$, respectively. The corresponding source rates are $x_{1}^{0}=5.3 \mathrm{Mb} / \mathrm{s}$ and $x_{2}^{0}=4.3 \mathrm{Mb} / \mathrm{s}$, respectively.

We first fix the capacities of the second and the third link to $10 \mathrm{Mb} / \mathrm{s}$ each. Algorithm 1 is tested for convergence as the capacity of the first link is varied. It can be verified from Fig. 9 that the convergence is observed only when the capacity of the first link is at least $10.5 \mathrm{Mb} / \mathrm{s}$. The capacity of the first link needed for convergence can be reduced to the minimum possible value by increasing the capacities of the other two links indefinitely, which results in vanishing prices on these links. The minimum required capacity of the first link becomes $x_{1}^{0}+x_{2}^{*}\left(q_{1}^{0}\right)=$ $9.9 \mathrm{Mb} / \mathrm{s}$ in this case. The minimum cannot be reduced all the way to $x_{1}^{0}+x_{2}^{0}=9.6 \mathrm{Mb} / \mathrm{s}$ since the two sigmoidal sources share the same link and must see the same price when the prices on other links go down to zero.

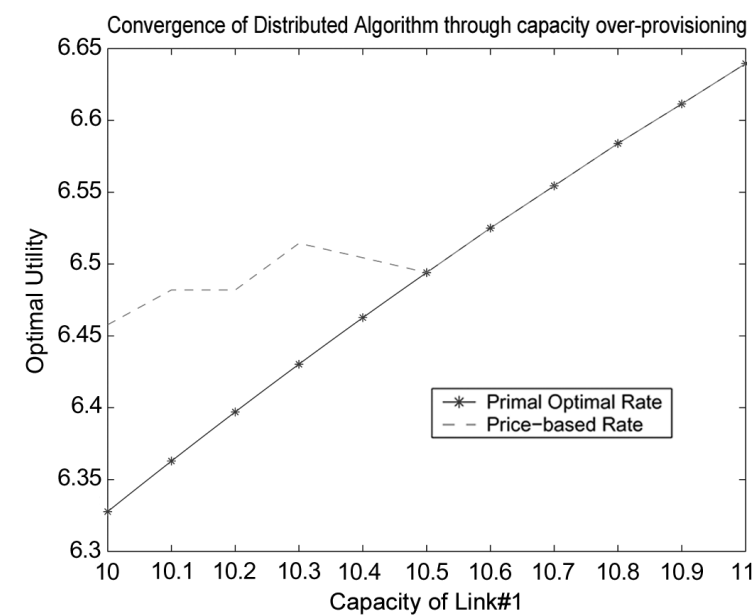

Fig. 9. (Example 4) Distributed Algorithm 1's convergence against link capacity $c$.

\section{Minimal Capacity}

As can be inferred from Theorem 3 , by selecting a sufficiently small price $\mathbf{p}$, we can push the optimal set of prices $\boldsymbol{\lambda}^{\mathrm{opt}}$ to a range where all the sigmoidal sources see a price less than the critical value. However, if the price $\mathbf{p}$ is not carefully selected, this could result in unnecessary over-provisioning of capacity. It is desirable to reduce the price $\mathbf{p}$ only to the extent required resulting in a capacity vector $\mathbf{c}$ that is on the Pareto-minimal boundary of $\mathcal{C}_{s}$, which we refer to as the minimal capacity vector $\mathbf{c}_{\min }(\mathbf{p})$.

The set of prices $\mathbf{p}$ that lead to a minimal capacity vector can be characterized under two distinct cases. If $\mathbf{R}_{n}$ is full column rank, then any pricing vector $\mathbf{p}$ that satisfies $\mathbf{q}_{n}^{0}=\mathbf{R}_{n}^{T} \mathbf{p}$ leads to a Pareto-minimal capacity vector

$$
\mathbf{c}_{\min }(\mathbf{p})=\mathbf{R}_{n} \mathbf{x}_{n}^{*}(\mathbf{p})+\mathbf{R}_{c} \mathbf{x}_{c}^{*}(\mathbf{p})
$$

where $\mathbf{p}$ satisfies the following equality:

$$
\mathbf{q}_{n}^{0}=\mathbf{R}_{n}^{T} \mathbf{p}
$$

If, on the other hand, $\mathbf{R}_{n}$ is not full row rank, then there might not exist any $\mathbf{p}$ such that $\mathbf{q}_{n}^{0}=\mathbf{R}_{n}^{T} \mathbf{p}$. Any price vector $\mathbf{p}$ such that for each $i$ there is some $j$ satisfying $\left\{\left(\mathbf{R}_{n}^{T} \mathbf{p}\right)_{j}=q_{j}^{0}, R_{i j} \neq\right.$ $0\}$ results in a Pareto-minimal capacity vector, $\mathbf{c}_{\min }$

$$
\mathbf{c}_{\min }(\mathbf{p})=\mathbf{R}_{n} \mathbf{x}_{n}^{*}(\mathbf{p})+\mathbf{R}_{c} \mathbf{x}_{c}^{*}(\mathbf{p})
$$

where $\mathbf{p}$ satisfies the following inequality:

$$
\mathbf{q}_{n}^{0} \succeq \mathbf{R}_{n}^{T} \mathbf{p}
$$

and for all $i$, there is a $j$ such that $\left\{\left(\mathbf{R}_{n}^{T} \mathbf{p}\right)_{j}=q_{j}^{0}, R_{i j} \neq 0\right\}$.

Minimal capacity vector may not be unique and is only minimal in the Pareto-optimal sense: it is impossible to find another pricing vector that satisfies the constraint and also leads to a different capacity vector each of whose component is smaller than that in $\mathbf{c}_{\min }(\mathbf{p})$.

Let $\mathcal{C}_{z}$ denote the set of minimum capacity vectors. Then $\mathcal{C}_{z}$ is the Pareto-minimal boundary of the set $\mathcal{C}_{s}$. To guarantee both convergence of Algorithm 1 and nonzero rate allocation to sigmoidal utility sources, it is sufficient to provision capacity to a 
TABLE II

Convergence of Algorithm 1 at DifFERENT CAPACITy Configurations

\begin{tabular}{|l|l|}
\hline Algorithm 1 converges, sigmoidal utility sources attain zero rate & $\mathbf{c} \in \mathcal{C}_{n}$ \\
\hline Algorithm 1 converges, sigmoidal utility sources attain non-zero rate & $\mathbf{c} \in \mathcal{C}_{s}$ \\
\hline Algorithm 1 convergence uncertain but duality gap is zero & $\mathbf{c} \in \mathcal{C}_{z}$ \\
\hline Algorithm 1 convergence uncertain & $\mathbf{c} \notin\left\{\mathcal{C}_{n} \cup \mathcal{C}_{z} \cup \mathcal{C}_{z}\right\}$ \\
\hline
\end{tabular}

value just a little more than the minimum capacity vector, since $\mathcal{C}_{s} \cap \mathcal{C}_{z}=\emptyset$. In fact, we see from (8) and (9) that $\mathcal{C}_{z}$ is the set of capacities at which at least one of the sigmoidal utility encounters it's critical price and thus convergence of Algorithm 1 is not assured although zero duality gap is achieved.

Thus far in the paper, we have quantified several relationships between the properties of Algorithm 1 and link capacity configurations, as summarized in Table II.

It is natural to further optimize the capacity configuration by associating a cost of $w_{l}$ for provisioning one unit of capacity on link $l$. We can then seek the capacity provisioning that minimizes the total cost $\sum_{l} w_{l} c_{l}$ and still results in convergence of Algorithm 1. The following capacity provisioning problem is thus formulated:

$$
\begin{array}{ll}
\text { minimize } & \sum_{l} w_{l} c_{l} \\
\text { subject to } & \mathbf{c}=\mathbf{R}_{n} \mathbf{x}_{n}^{*}(\mathbf{p})+\mathbf{R}_{c} \mathbf{x}_{c}^{*}(\mathbf{p}) \\
& \mathbf{q}_{n}^{0} \succ \mathbf{R}_{n}^{T} \mathbf{p} .
\end{array}
$$

However, problem (10) is a non-convex optimization problem because the constraint set is nonconvex (due to $x_{s}^{*}(\mathbf{p})$ for $s \in$ $\left.S_{n}\right)$.

\section{Capacity Provisioning for a Medium Size Network}

Example 5: Consider a medium size network as shown in Fig. 10, consisting of 10 flows that share 8 links. Let the first 5 flows be elastic with concave utilities and the next 5 inelastic with sigmoidal utility functions. We assume that all links in the network have the same capacity $c$, and examine the convergence of Algorithm 1 as $c$ is varied.

In Fig. 11, we plot the price-based rate allocation achieved by Algorithm 1 when the link capacities are all set to $c=10 \mathrm{Mb} / \mathrm{s}$. The price-based rate allocation is plotted on the top half for elastic sources and the on the bottom half for inelastic sources. We notice that the elastic sources achieve convergence eventually while only one of the inelastic sources achieves convergence. Indeed, the capacity vector $\mathbf{c}$ all of whose components are equal to $10 \mathrm{Mb} / \mathrm{s}$ does not satisfy the criterion specified in Theorem 3 . We also observe that the link prices (not plotted) converge even though the source rates do not. Furthermore, the sources (both elastic and inelastic ones) whose rates achieve convergence do not necessarily converge to the globally optimal rates.

Figs. 12 and 13 show the evolutions of price-based rate allocation when the link capacities are all equal to 15 and $20 \mathrm{Mb} / \mathrm{s}$, respectively. At $c=15 \mathrm{Mb} / \mathrm{s}$, elastic sources converge faster than in Fig. 11, and four out of the five inelastic sources converge. In fact, except for the inelastic source that does not converge, the path prices seen by all other sources converge to values less than the critical slopes $q_{s}^{0}$. When link capacities are all set to $c=20 \mathrm{Mb} / \mathrm{s}$, all sources achieve rate convergence and the convergence is faster as well. It can be verified that the path prices indeed converge to values less than the critical $q_{s}^{0}$ for all the inelastic sources.

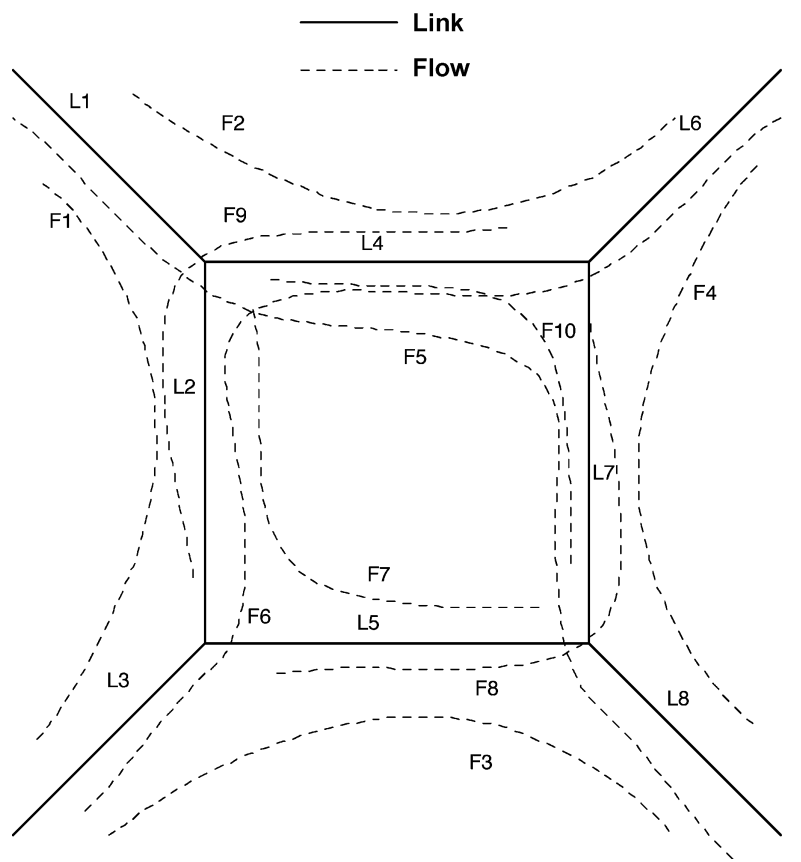

Fig. 10. Network topology for Example 5.
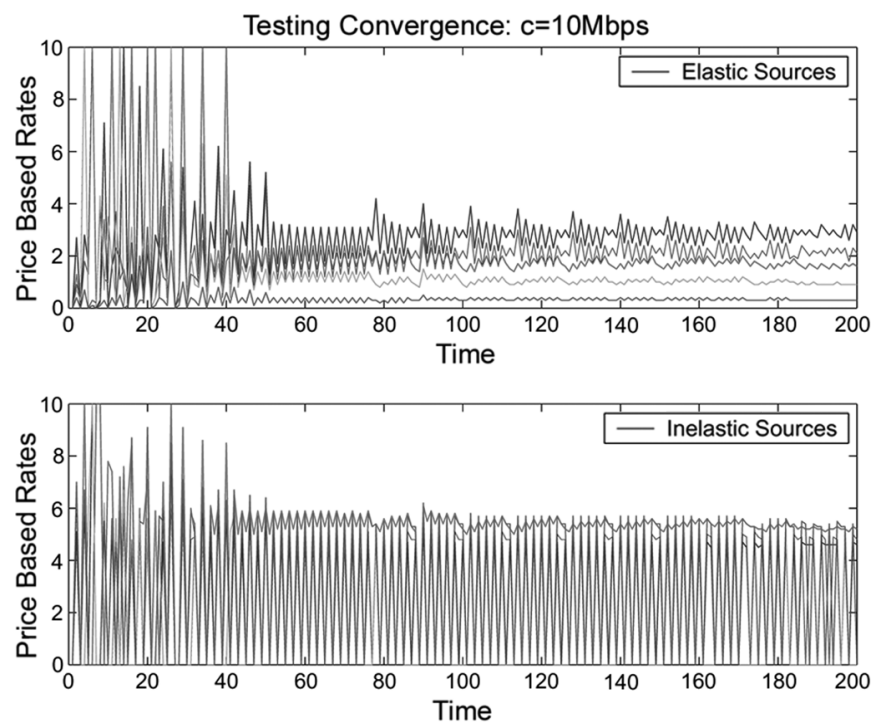

Fig. 11. (Example 5) Distributed Algorithm 1's convergence when link capacities $=10 \mathrm{Mb} / \mathrm{s}$. Four of the five inelastic flows' rates do not converge.

Some observations for this example are in order. First, link prices $\left\{\lambda_{l}\right\}$ and hence path prices $\left\{q_{s}\right\}$ always converge. Path prices $\left\{q_{s}\right\}$ converge to the critical slope $\left\{q_{s}^{0}\right\}$ for the inelastic sources that do not achieve convergence in rate, and to a value less than the critical slope $\left\{q_{s}^{0}\right\}$ for the inelastic sources that do converge. Second, price-based rate allocation always converges for elastic sources, but converges for inelastic sources only if 

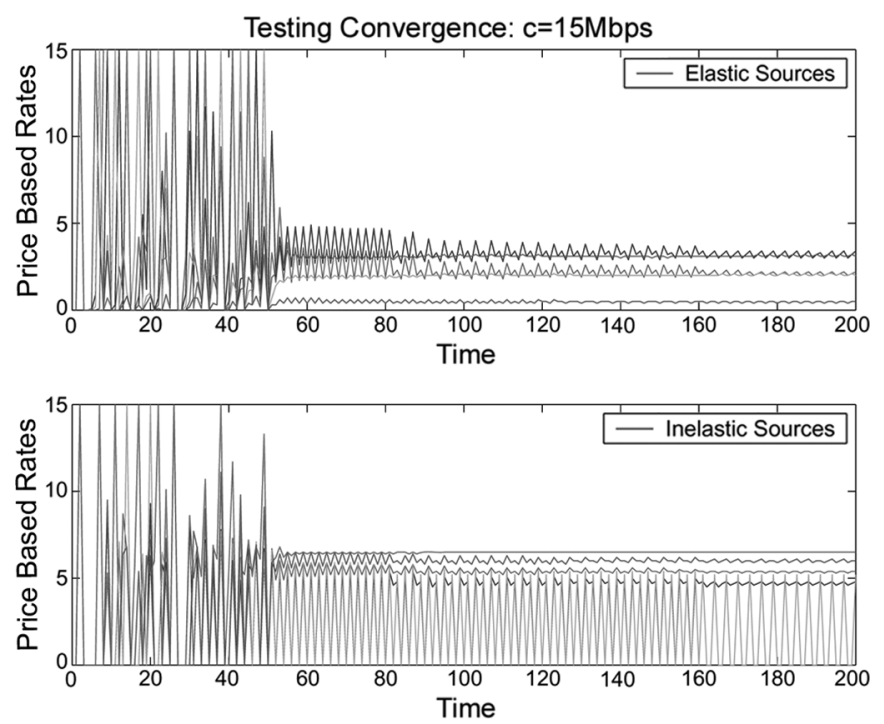

Fig. 12. (Example 5) Distributed Algorithm 1's convergence against link capacities $=15 \mathrm{Mb} / \mathrm{s}$. One of the five inelastic flows' rates does not converge.
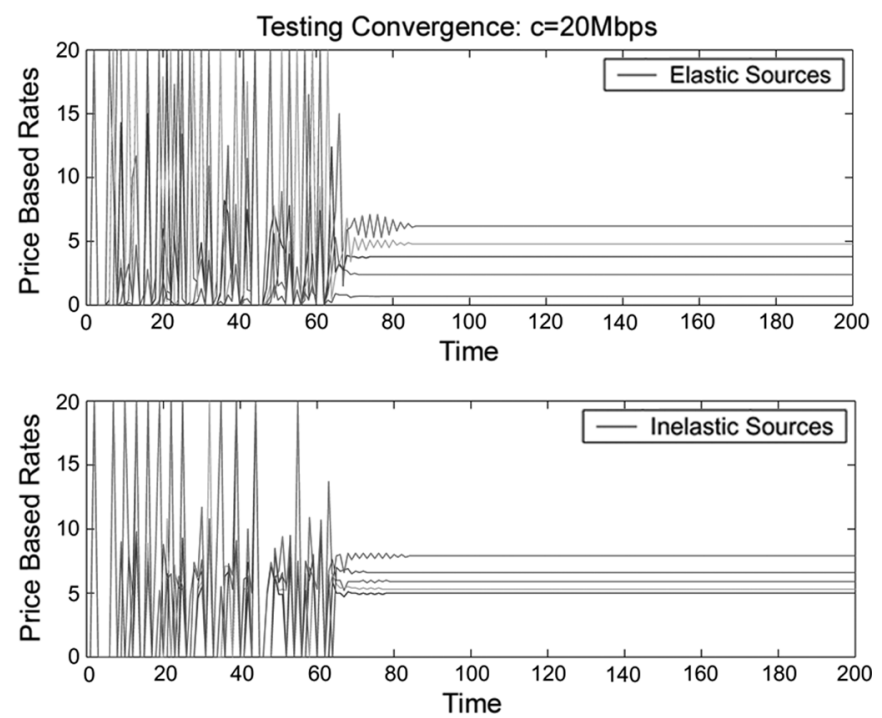

Fig. 13. (Example 5) Distributed Algorithm 1's convergence against link capacities $=20 \mathrm{Mb} / \mathrm{s}$. All source rates converge, and to the globally optimal allocation.

the link capacities are sufficiently high. Third, when all sources achieve convergence, the convergence is to the primal optimum. However, when only some of the sources achieve convergence, the converged solution may not be a global optimum.

\section{Discontinuous Utilities: OPTIMIZATION FRAMEWORK}

\section{A. NUM Formulation and Capacity Provisioning}

In this section, we turn to real-time flows, whose utilities are best represented as discontinuous functions, and consider real-time flows sharing link capacity with elastic flows. We use $i$ to index a total of $S_{c}$ elastic flows with concave utilities, and $j$ to index a total of $S_{r}$ real-time flows with discontinuous utilities. A flow $j$ from real-time IP applications or CBR ATM applications requires a constant playback rate $v_{j}$, attaining zero utility if its allocated rate is below threshold $v_{j}$ and a constant positive utility $U_{j}^{r}$ (or a concave increasing utility under a different modeling assumption in Sections IV-C) if its rate is at or above $v_{j}$. This model can be generalized to utility functions in stair-case shapes with multiple discontinuous jumps, and the methodologies developed in this section can still be applied.

As discussed in [18], for such real-time flows, admission control is needed. For each source $j$, an admission decision $a_{j}$ is made. Utility obtained is a constant $U_{j}^{r}$ if $a_{j}=1$ (i.e., the flow is admitted) and 0 if $a_{j}=0$ (i.e., the flow is rejected). Notice that the optimization variables for flows indexed by $j$ are boolean: $a_{j} \in\{0,1\}$, rather than continuous.

Now consider a NUM formulation for rate allocation in a network shared by both types of flows, with the set of links used by a concave utility flow $i$ denoted as $L_{c}(i)$ and that used by a real-time flow $j$ denoted as $L_{r}(j)$

$$
\begin{array}{ll}
\operatorname{maximize} & \sum_{i} U_{i}\left(x_{i}\right)+\sum_{j} a_{j} U_{j}^{r} \\
\text { subject to } & \sum_{i: l \in L_{c}(i)} x_{i}+\sum_{j: l \in L_{r}(j)} a_{j} v_{j} \leq c_{l}, \quad \forall l, \\
& x_{i} \geq 0, \forall i, \\
& a_{j} \in\{0,1\}, \forall j
\end{array}
$$

where the optimization variables are $\left\{x_{i}\right\}$ and $\left\{a_{j}\right\}$.

Two natural questions follow: under what special conditions on link capacity vector $\mathbf{c}$ will the optimal solution be admitting all (or none) of the real-time flows? And in general how should admission control be carried out distributively and jointly with price-based rate allocation for elastic flows? These two questions are answered in this and next subsections, respectively.

Problem (11) is in general an intractable, integer-constrained optimization. However, we can gain insight on the optimal solution by studying a relaxed version of the problem, where we relax variables $\left\{a_{j}\right\}$ to take any value in the interval $[0,1]$ :

$$
\begin{array}{ll}
\operatorname{maximize} & \sum_{i} U_{i}\left(x_{i}\right)+\sum_{j} a_{j} U_{j}^{r} \\
\text { subject to } & \sum_{i: l \in L_{c}(i)} x_{i}+\sum_{j: l \in L_{r}(j)} a_{j} v_{j} \leq c_{l}, \forall l, \\
& x_{i} \geq 0, \forall i, \\
& 0 \leq a_{j} \leq 1, \forall j .
\end{array}
$$

The relaxed problem (12) is an efficiently-solvable convex optimization problem, since the relaxed constraint region is now convex and the function to be maximized is concave. We have the following characterization of the relationship between the optimal (relaxed) admission variable $a_{j}^{\text {opt }}$ and the optimal path price $q_{j}^{\mathrm{opt}}=\sum_{l \in L_{r}(j)} \lambda_{j}$ seen by source $j$.

Theorem 4: Each optimum admission variable $a_{j}^{\text {opt }}$ for problem (12) satisfies the following conditions:

$$
\begin{array}{ll}
\mathrm{A} 1: & a_{j}^{\mathrm{opt}}=1 \Rightarrow U_{j}^{r} / v_{j} \geq q_{j}^{\mathrm{opt}} \\
\mathrm{A} 2: & 0<a_{j}^{\mathrm{opt}}<1 \Rightarrow U_{j}^{r} / v_{j}=q_{j}^{\mathrm{opt}} \\
\mathrm{A} 3: & a_{j}^{\mathrm{opt}}=0 \Rightarrow U_{j}^{r} / v_{j} \leq q_{j}^{\mathrm{opt}} \\
\mathrm{B} 1: & U_{j}^{r} / v_{j}>q_{j}^{\mathrm{opt}} \Rightarrow a_{j}^{\mathrm{opt}}=1 \\
\mathrm{~B} 2: & U_{j}^{r} / v_{j}=q_{j}^{\mathrm{opt}} \Rightarrow 0 \leq a_{j}^{\mathrm{opt}} \leq 1 \\
\mathrm{~B} 3: & U_{j}^{r} / v_{j}<q_{j}^{\mathrm{opt}} \Rightarrow a_{j}^{\mathrm{opt}}=0
\end{array}
$$

where $q_{j}{ }^{\text {opt }}$ is the optimal dual variable of problem (12). 
Proof: We begin with the Lagrangian of (12).

$$
\begin{aligned}
L(\mathbf{x}, \mathbf{a}, \boldsymbol{\lambda}, \boldsymbol{\gamma}, \boldsymbol{\nu})= & \sum_{i} U_{i}\left(x_{i}\right)+\sum_{j} a_{j} U_{j}^{r} \\
& +\sum_{l} \lambda_{l}\left(c_{l}-\sum_{i: l \in L_{c}(i)} x_{i}-\sum_{j: l \in L_{r}(j)} a_{j} v_{j}\right) \\
& +\sum_{j} \gamma_{j}\left(1-a_{j}\right)+\sum_{j} \nu_{j} a_{j}
\end{aligned}
$$

where $\boldsymbol{\lambda} \succeq 0$ has the same link price interpretation as before and $\boldsymbol{\gamma} \succeq 0$ and $\boldsymbol{\nu} \succeq 0$ are the prices associated with the constraints on $a_{j}$. Let $\left\{\boldsymbol{\lambda}^{\mathrm{opt}}, \boldsymbol{\gamma}^{\mathrm{opt}}, \boldsymbol{\nu}^{\mathrm{opt}}\right\}$ denote the optimal dual prices and $\left\{\mathbf{x}^{\mathrm{opt}}, \mathbf{a}^{\mathrm{opt}}\right\}$ the optimal primal variables. Complementary slackness condition for primal-dual optimality [3] imposes the following optimality conditions on the real-time flows:

$$
\begin{aligned}
U_{j}^{r} & =q_{j}^{\mathrm{opt}} v_{j}+\gamma_{j}^{\mathrm{opt}}-\nu_{j}^{\mathrm{opt}} \\
\gamma_{j}^{\mathrm{opt}}\left(1-a_{j}^{\mathrm{opt}}\right) & =0 \\
\nu_{j}^{\mathrm{opt}} a_{j}^{\mathrm{opt}} & =0
\end{aligned}
$$

where $q_{j}=\sum_{l \in L_{r}(j)} \lambda_{l}$. If $a_{j}^{\text {opt }}=1$, then $\gamma_{j}^{\text {opt }} \geq 0$ and $\nu_{j}^{\text {opt }}=0$, resulting in $U_{j}^{r} / v_{j} \geq q_{j}^{\text {opt }}$. If $0<a_{j}^{\text {opt }}<1$, then $\gamma_{j}^{\text {opt }}=0$ and $\nu_{j}^{\text {opt }}=0$, resulting in $U_{j}^{r} / v_{j}=q_{j}^{\text {opt }}$. If $a_{j}^{\text {opt }}=0$, then $\gamma_{j}^{\text {opt }}=0$ and $\nu_{j}^{\text {opt }} \geq 0$, resulting in $U_{j}^{r} / v_{j} \leq q_{j}^{\text {opt }}$.

To prove the converse part, note that $\gamma_{j}^{\text {opt }} \neq 0$ and $\nu_{j}^{\text {opt }} \neq$ 0 cannot both be 0 at the same time since that requires both $a_{j}^{\text {opt }}=1$ and $a_{j}^{\text {opt }}=0$. If $U_{j}^{r} / v_{j}>q_{j}^{\text {opt }}$ we have $\gamma_{j}^{\text {opt }}>0$ and $\nu_{j}^{\text {opt }}=0$, thus $a_{j}^{\text {opt }}=1$. If $U_{j}^{r} / v_{j}=q_{j}^{\text {opt }}$, then $\gamma_{j}^{\text {opt }}=0$ and $\nu_{j}^{\text {opt }}=0$, thus $0 \leq a_{j}^{\text {opt }} \leq 1$. If $U_{j}^{r} / v_{j}<q_{j}^{\text {opt }}$, then $\gamma_{j}^{\text {opt }}=0$ and $\nu_{j}^{\text {opt }}>0$, thus $a_{j}^{\text {opt }}=0$.

The original NUM problem (11) has an optimal network total utility that is upper bounded by the optimal network utility in the relaxed version of the problem (12), because the constraint set of the relaxed version subsumes the constraint set of the original problem. This means that, when the optimal solution for the relaxed version of the problem is achieved at the extreme points $\left\{a_{j}=0, a_{j}=1\right\}$, which fall within the constraint set of the original problem, the solution is optimal for the original problem as well. Therefore, the cases of B1 and B3 in the above Theorem are the most useful ones.

Consider now the possibility of admitting all the real-time flows and allocating rates to elastic flows by Algorithm 1, as long as link capacities fall within certain sets. Denote the constant ratio $U_{j}^{r} / v_{j}$ as $q_{j}^{r}$ for real-time flow $j$. Note that $q_{j}^{r}$ is a constant parameter and not a function of link price vector $\lambda$. We can admit every real-time flow into the network if $q_{j}^{r}>q_{j}^{\text {opt }}$. This is similar to the discussion in Section III where we discuss the minimal link capacity provisioning required for applicability of Algorithm 1 for rate allocation among nonconcave utility flows. Indeed, a similar argument proves the following theorem. Let $\mathbf{R}_{r}$ be the routing matrix for the real-time flows, $\mathbf{v}$ be the vector of constant rates $v_{j}$ of the real-time flows, $\mathbf{R}_{c}$ be the routing matrix of the elastic sources, and $\mathbf{q}_{r}$ be the vector whose $j$ th entry is $q_{j}^{r}$.

Theorem 5: The optimal solution to problem (11) is to admit all real-time flows, i.e., $a_{j}^{\text {opt }}=1, \forall j$, if there is a $\mathbf{p} \succeq 0$ satisfying the following inequality:

$$
\mathbf{q}_{r} \succeq \mathbf{R}_{r}^{T} \mathbf{p}
$$

and the link capacities $\mathbf{c}=\mathbf{c}(\mathbf{p})$ where

$$
\mathbf{c}(\mathbf{p})=\mathbf{R}_{r} \mathbf{v}+\mathbf{R}_{c} \mathbf{x}_{c}^{*}(\mathbf{p}) .
$$

Remark 4: If the capacity vector is such that

$$
\begin{aligned}
\mathbf{c} & \prec \mathbf{R}_{c} \mathbf{x}_{c}^{*}(\mathbf{p}) \text { for some } \mathbf{p} \text { that satisfies : } \\
\mathbf{q}_{r} & \prec \mathbf{R}_{r}^{T} \mathbf{p}
\end{aligned}
$$

then again Algorithm 1 will find the globally optimal rate allocation for all elastic flows. But in this uninteresting case, inelastic sources will receive zero rate allocation and none of the real-time applications are admitted in the optimal solution.

\section{B. Price-Based Admission Control Heuristics}

Let $\mathcal{C}_{\text {all }}$ and $\mathcal{C}_{\text {none }}$ denote the set of link capacity vectors that satisfy (15) and (16), respectively. If the capacity vector c is such that it is neither in $\mathcal{C}_{\text {all }}$ nor in $\mathcal{C}_{\text {none }}$, then the optimal admission decision for problem (11) is uncertain and the outcome of Algorithm 1 unclear. In such cases, as well as in cases where a priori determination of whether $\mathbf{c}$ falls in $\mathcal{C}_{\text {all }}$ or $\mathcal{C}_{\text {none }}$ is impossible, price-based admission control policy needs to be implemented, preferably at the network edge in a distributed way.

Inspired by the results in the previous subsection and conditions B1 and B3 in Theorem 4, we propose the following price-based admission control heuristic. This heuristic is conducted locally at the edge by each real-time source, following the end-to-end principle and assuming cooperative end users. It is parameterized by nonnegative integers $m$ and $n$.

Algorithm 3-Two-Phase Admission Control Heuristics: If the price $q_{j}=\sum_{l \in L_{r}(j)} \lambda_{j}$ seen by a real-time source $j$ is smaller than the constant ratio $U_{j}^{r} / v_{j}$ for $m$ time slots, it is tentatively admitted, and a message is passed to reserve $v_{j}$ amount of bandwidth along the route it uses. This tentative admission period is the resource reservation phase (the first phase).

If the price $q_{j}$ continues to be smaller than $U_{j}^{r} / v_{j}$ for $n$ more time slots (the second phase), the flow is formally admitted and transmission can start, otherwise the flow is rejected and has to wait for another window of $m$ times slots, during which the price is sufficiently low, before entering the resource reservation phase again.

Remark 5: As is typical with other price-based heuristics (e.g., [11]), Algorithm 3 is in general suboptimal, with larger waiting parameters $(m, n)$ enhancing the probability that the correct admission decision is made but also increasing the latency incurred.

Example 6.: The admission control heuristic is tested on various networks. Typical results are summarized below for the network shown in Fig. 14 with three links and four flows. Link capacities are 30,20, and $40 \mathrm{Mb} / \mathrm{s}$, respectively. Flows 1 and 2 are elastic data flows, with utility functions $U_{i}=\log \left(1+x_{i}\right)$, $i=1,2$, while flows 3 and 4 are inelastic real-time flows with $\bar{U}_{3}=1, \bar{U}_{4}=0.2$, and playback rates of $v_{3}=5, v_{4}=7$. If both flows 3 and 4 use $(m, n)=(8,8)$ in the admission control heuristic, Fig. 15 shows the resulting rate allocation iterations and convergence to the optimal solution. In this example, flow 3 is admitted in the first try (passing through both phases), and flow 4 only enters the resource reservation phase once, during which it is rejected. The optimal solution for this utility maximization problem is indeed to admit flow 3 and reject flow 4 . 


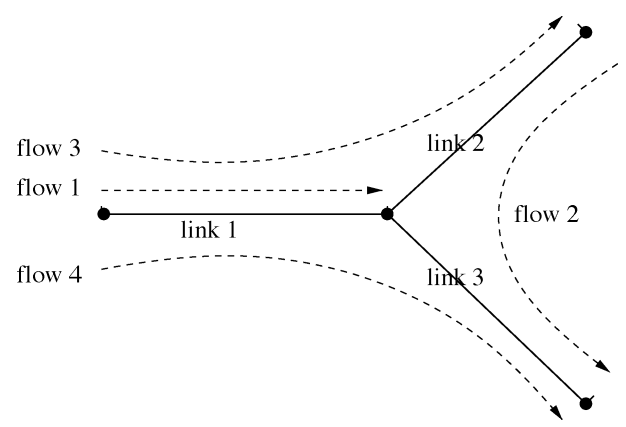

Fig. 14. Network topology for Example 6.

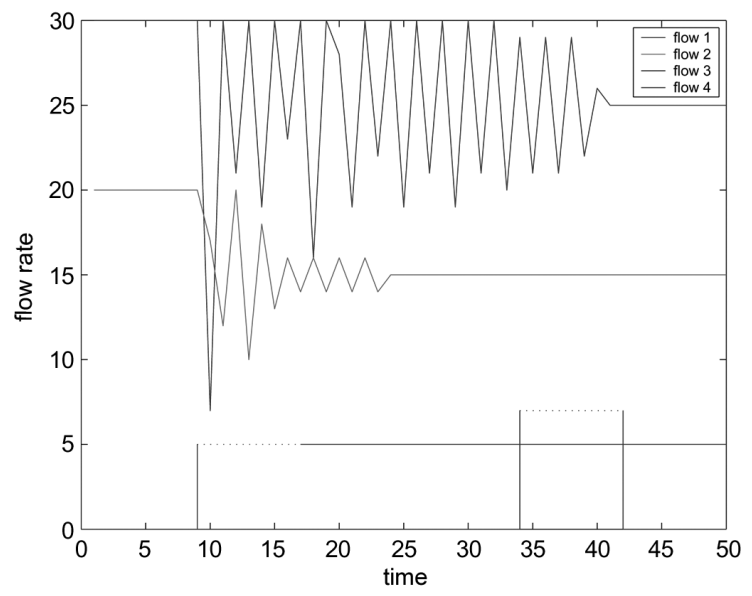

Fig. 15. (Example 6) Rate allocation based on the admission control heuristic.

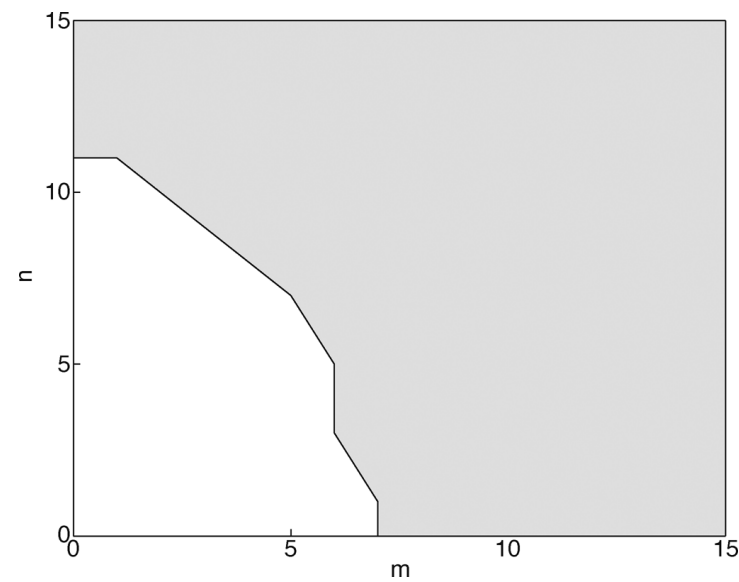

Fig. 16. (Example 6) The correct decision region in the $(m, n)$ plane is shaded.

Extensive simulations are conducted on the same topology for other $(m, n)$ pairs. A variety of rate allocation scenarios are observed and classified into correct or incorrect final admission decisions by comparing against the global optimum computed through exhaustive search. The region in the $(m, n)$ plane where correct decisions are made is the shaded region (and the rest of the 2-D plane upwards and to the right) in Fig. 16. This connected region illustrates the following desirable and intuitive properties of the heuristic.

- When either $m$ or $n$ is larger than a threshold $m_{0}$ or $n_{0}$, the other parameter can be as small as zero. If both $m$ and $n$ are nonzero, they can be smaller than $m_{0}$ or $n_{0}$ and still remain in the correct decision region.

- The Pareto optimal tradeoff curve between minimizing $n$ and minimizing $m$ is the boundary line between the shaded region and the unshaded region. If the total latency before formal admission needs to be minimized, it is best to operate at the point ( $m=7, n=0)$ for the network in Fig. 14 .

- In practice, it is unlikely that the best $(m, n)$ will be used. Thus, it is useful to observe that the latency associated with any point on the Pareto optimal tradeoff curve in the $(m, n)$ plane is only about $20 \%$ of the time it takes for all the flows to converge to very close to the optimum. This shows the effectiveness of this heuristic in reducing the time it takes to make the right admission decision.

\section{Rate Allocation Among Different Groups of Flows}

We conclude this section on real-time flows with a useful variant of discontinuous utility model. Modeling the situation where rates larger than $v_{j}$ may lead to improvements in user perception of the real-time applications, we now allow the utility functions to grow beyond $U_{j}^{r}$, following a concave utility $U_{j}\left(x_{j}\right)$ with $U_{j}\left(v_{j}\right)=U_{j}^{r}$. We refer to these flows, still indexed by $j$, as enhanced real-time flows. An example of such utilities is shown as type (c) utility curve in Fig. 1.

Consider the case where link capacity vector belongs to $\mathcal{C}_{\text {all }}$ as in Theorem 5. We may admit all real-time flows and ensure that their rates are never smaller than the necessary threshold $v_{j}$. However, these inelastic flows do not back-off their rates below $v_{j}$ while at the same time compete with elastic flows for the remaining capacity $c_{l}-\sum_{j: l \in L_{r}(j)} v_{j}$. Below, we propose a mechanism to control fairness of rate allocation between real-time and elastic flows beyond that provided by the utility functions and a hard bandwidth sharing constraint. We limit the allocation to the group of enhanced real-time flows to a fraction $\theta_{l}$ of total link capacity $c_{l}$ of link $l$. We assume that $\theta_{l}$ is such that $\theta_{l} c_{l}$ is large enough to admit all the enhanced real-time flows sharing link $l$. The resulting problem of rate allocation among these two groups of flows is thus formulated as follows:

$$
\begin{aligned}
\text { maximize } & \sum_{i} U_{i}\left(x_{i}\right)+\sum_{j} U_{j}\left(x_{j}\right) \\
\text { subject to } & \sum_{i: l \in L_{c}(i)} x_{i}+\sum_{j: l \in L_{r}(j)} x_{j} \leq c_{l}, \forall l, \\
& \sum_{j: l \in L_{r}(j)} x_{j} \leq \theta_{l} c_{l}, \forall l, \\
& x_{j} \geq v_{j}, \forall j \\
& \mathbf{x} \succeq 0 .
\end{aligned}
$$

The above problem can be solved for globally optimal rate allocation by the following.

Algorithm-Rate Allocation Among Groups: This algorithm is Algorithm 1 together with two modifications.

- At each iteration $t$, the allocated rate to a source in the enhanced real-time group is projected onto the interval $\left[v_{j}, \min _{l \in L_{r}(j)} c_{l}\right]$, i.e., if $x_{j}(t)<v_{j}$ at some iteration $t$, set $x_{j}(t)=v_{j}$.

- Distributively on each link, update another link price vector called "fair share prices" $\boldsymbol{\sigma}$, which enforces the constraint 


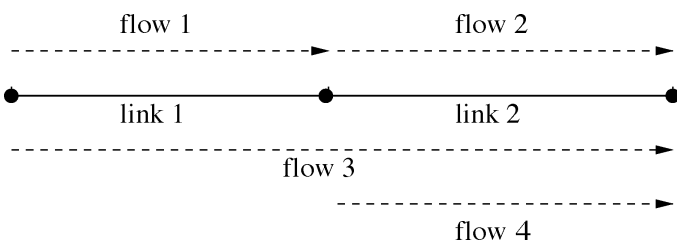

Fig. 17. Network topology for Example 7.

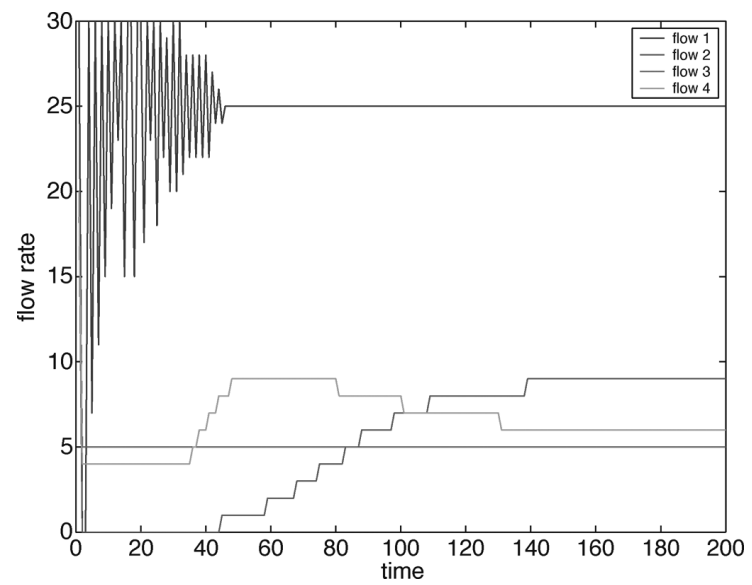

Fig. 18. (Example 7) Source rate allocation among four sources in two groups.

that at least $\theta_{l}$ percent of link capacity on link $l$ is provided for elastic flows

$$
\sigma_{l}(t+1)=\left[\sigma_{l}(t)-\alpha(t)\left(\theta_{l} c_{l}-\sum_{j: l \in L_{r}(j)} x_{j}^{*}(t)\right)\right]^{+}
$$

where step size $\alpha(t)$ can be taken as $\beta / t$ for some constant $\alpha_{0}>0$.

Each source in the enhanced real-time group uses the total price $q_{j}=\sum_{l \in L_{r}(j)}\left(\lambda_{l}+\sigma_{l}\right)$ (the sum of congestion price and fair share price). Each source in the elastic group uses price $q_{i}=$ $\sum_{l \in L_{c}(i)} \lambda_{l}$

Theorem 6: Algorithm 4 converges to the globally optimal rate allocation of (17).

Proof: Problem (17) is a convex optimization problem with zero duality gap. Skipping details of the derivation due to space limitation, Algorithm 4 can be verified to be a subgradient method solving the Lagrange dual problem. The proof of convergence is then a direct result of the convergence of subgradient algorithms [1].

Example 7: A typical simulation for Algorithm 4 is shown for the network in Fig. 17, where link capacities are 30 and $20 \mathrm{Mb} / \mathrm{s}$, respectively. Flows 3 and 4 are the enhanced real-time flows with utility functions $U_{3}=0.871 x_{3}^{0.1}, U_{4}=4.1808 x_{4}^{0.9}$ pass the threshold playback rate of $v_{3}=5, v_{4}=4 \mathrm{Mb} / \mathrm{s}$. Flows 1 and 2 are elastic data flows with utility functions $U_{i}=\log \left(1+x_{1}\right)$, $i=1,2$.

Fig. 18 shows the source rate iterations. It is observed that the two enhanced real-time flows never have their source rates dropped below the minimum thresholds, and, at the same time, they do not occupy more than $\theta_{1}=30 \%, \theta_{2}=50 \%$ of link capacities. The equilibrium rate allocation for flow 4 at global optimality is six units, more than the minimum threshold $v_{4}$. The equilibrium in this numerical experiment has been verified to be the globally optimal rate allocation through exhaustive search.

\section{CONCLUding REMARKS}

The standard distributed subgradient algorithm (Algorithm 1) for network utility maximization has been effective in modeling TCP congestion control mechanisms and in allocating rates among elastic flows. However, many flows are inelastic and their rate allocation remains an open problem. This paper tackles the difficult problems in distributed solution for network utility maximization without the widespread, yet often invalid assumptions of concave and continuous utility functions.

We first examine the case where some utilities are general nonlinear, nonconcave functions, and show that Algorithm 1 can still converge to the globally optimal rate allocation. A sufficient condition to ensure the applicability of standard price-based rate allocation is the continuity of $\mathbf{x}^{*}(\boldsymbol{\lambda})$ at optimal prices $\boldsymbol{\lambda}^{\mathrm{opt}}$. This conclusion motivates the study of the relationships between link capacity provisioning and convergence of Algorithm 1, and the results are summarized in Table II. In particular, we derive the link capacity vectors that guarantee the convergence and optimality of Algorithm 1 when the nonconcavities are sigmoidal, and quantify the tradeoff between the cost of provisioning link capacities and the applicability of price-based rate allocation for inelastic flows.

We then study the case where real-time flows with discontinuous utility functions (regulated by admission control) share a network with elastic flows (regulated by rate control). We provide a sufficient condition on link capacity configuration so that all real-time flows can be admitted. When such conditions do not hold, we propose a price-based distributed admission control heuristic (Algorithm 3). We motivate a need to protect elastic flows' share of link capacity and develop an optimal algorithm (Algorithm 4) to allocate rates among elastic flows and enhanced real-time flows.

The results in Section II are also applicable to general problems of convex constrained, nonconcave maximization with a separable objective function, such as spectrum management in OFDM communication systems and wireless network power control in low SIR regime.

As one of the few papers studying rate allocation among inelastic flows in a rigorous manner, this paper's contribution is summarized in Table I. Given that these network utility maximization problems are NP-hard, it is not surprising that many issues remain open on this challenging topic. First, it will be interesting to arrive at a set of sufficient and necessary conditions for the optimal convergence of the distributed subgradient algorithm that can be distributively calculated at each source to decide independently whether or not to participate in the pricebased rate allocation. Second, bounds on suboptimality gap for our price-based admission control heuristic are also highly desirable. Third, similar to most of the research literature on network utility maximization, we have assumed a fixed number of flows with infinite backlog. When utilities are concave and continuous, it is recently shown (e.g., in [13] and [20]) that Algorithm 1 remains stochastically stable when flows arrive at 
the network according to Poisson distribution with exponentially-distributed file sizes, even when time-scale separation (Algorithm 1 converges before the number of flows changes) is not assumed. When some flows have nonconcave utilities, we know that Algorithm 1 will still remain stochastically stable provided that the time-scale separation can be assumed and link capacity vector $\mathbf{c}$ satisfy the condition for Algorithm 1's convergence (in the deterministic model) in Theorem 3. This is because the optimal rate allocation will occur in the concave regions of the nonconcave utilities. However, Algorithm 1's stochastic stability remains unknown when neither concave utility assumption nor time-scale separation assumption holds. Furthermore, for general filesize distrobution, stochastic stability of concave utility maximization has recently been proved for certain utility functions, e.g., in [6], but the case for nonconcave utility functions remains unknown.

\section{ACKNOWLEDGMENT}

The authors would like to acknowledge very helpful discussions with J. W. Lee at Yonsei University, W. Y. at the University of Toronto, N. Shroff at Purdue University, V. Anantharam at UC Berkeley, D. Ming Chiu at the Chinese University of Hong Kong, S. Low, J. Doyle, and K. Tang at Caltech, and several reviewers.

\section{REFERENCES}

[1] D. P. Bertsekas, Nonlinear Programming. Belmont, MA: Athena Scientific, 1999.

[2] D. P. Bertsekas, A. Nedic, and A. E. Ozdaglar, "Min common max crossing duality: A simple geometric framework for convex optimization and minimax theory," Rep. LIDS-P-2536, Jan. 2002.

[3] S. Boyd and L. Vandenberghe, Convex Optimization. New York: Cambridge Univ. Press, 2004.

[4] M. Chiang, "Balancing transport and physical layers in wireless multihop networks: Jointly optimal congestion control and power control," IEEE J. Sel. Areas Commun., vol. 23, no. 1, pp. 104-116, Jan. 2005.

[5] M. Chiang, S. H. Low, A. R. Calderbank, and J. C. Doyle, "Layering as optimization decomposition: A mathematical theory of network architectures," Proc. IEEE, vol. 96, no. 1, pp. 255-312, Jan. 2007.

[6] M. Chiang, D. Shah, and A. Tang, "Stochastic stability of network utility maximization: General filesize distribution," in Proc. Allerton Conf., Sep. 2006.

[7] M. Chiang et al., "Power control by geometric programming," in IEEE Trans. Wireless Commun., to be published.

[8] M. Fazel and M. Chiang, "Nonconcave network utility maximization through sum of squares method," presented at the IEEE Control and Decision Conf., Seville, Spain, Dec. 2005.

[9] F. P. Kelly, A. Maulloo, and D. Tan, "Rate control for communication networks: Shadow prices, proportional fairness and stability," J. Opt. Res. Soc., vol. 49, no. 3, pp. 237-252, Mar. 1998.

[10] R. J. La and V. Anantharam, "Utility-based rate control in the Internet for elastic traffic," IEEE/ACM Trans. Netw., vol. 10, no. 2, pp. 272-286, Apr. 2002.

[11] J. W. Lee, R. R. Mazumdar, and N. Shroff, "Non-convex optimization and rate control for multi-class services in the Internet," presented at the IEEE INFOCOM, Hong Kong, China, Mar. 2004.

[12] S. H. Low, "A duality model of TCP and queue management algorithms," IEEE/ACM Tran. Netw., vol. 11, no. 4, pp. 525-536, Aug. 2003.

[13] X. Lin and N. B. Shroff, "On the stability region of congestion control," in Proc. Allerton Conf., 2004.

[14] L. Massoulie and J. W. Roberts, "Bandwidth sharing and admission control for elastic traffic," Telecommun. Syst., vol. 15, pp. 185-201, Mar. 2000.

[15] J. Mo and J. Walrand, "Fair end-to-end window-based congestion control," IEEE/ACM Trans. Netw., vol. 8, no. 5, pp. 556-567, Oct. 2000.
[16] R. T. Rockafellar, Network Flows and Monotropic Programming. Belmont, MA: Athena Scientific, 1998.

[17] R. T. Rockafellar, "Lagrange multipliers and optimality," SIAM Rev., vol. 35, pp. 183-283, 1993.

[18] S. Shenker, "Fundamental design issues for the future Internet," IEEE J. Sel. Areas Commun., vol. 13, no. 7, pp. 1176-1188, Sep. 1995.

[19] R. Srikant, The Mathematics of Internet Congestion Control. Boston, MA: Birkhauser, 2004.

[20] R. Srikant, "On the positive recurrence of a Markov chain describing file arrivals and departures in a congestion-controlled network," in Proc. IEEE Comput. Commun. Workshop, 2004.

[21] H. Yäiche, R. R. Mazumdar, and C. Rosenberg, "A game theoretic framework for bandwidth allocation and pricing of elastic connections in broadband networks: Theory and algorithms," IEEE/ACM Trans. Netw., vol. 8, no. 5, pp. 667-678, Oct. 2000 .

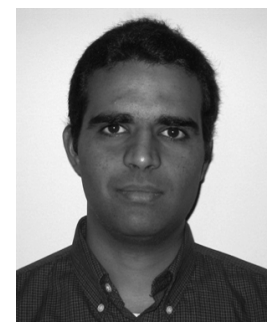

Prashanth Hande received the B.Tech. degree from the Indian Institute of Technology, Mumbai, India, in 1998 and the M.S. degree from Cornell University, Ithaca, NY, in 2000, both in electrical engineering. $\mathrm{He}$ is currently working toward the Ph.D. degree in the Department of Electrical Engineering, Princeton University, Princeton, NJ.

$\mathrm{He}$ is also a Staff Engineer at Qualcomm Flarion Technologies, Bedminster, NJ. His interests are in wireless communications, network architectures and broadband access networks.

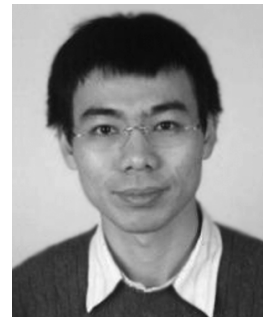

Shengyu Zhang received the B.S. degree in mathematics from Fudan University, Shanghai, China, in 1999, the M.S. degree from the Computer Science Department, Tsinghua University, Beijing, China, in 2002 (under the supervision of M. Ying), and the Ph.D. degree from the Computer Science Department, Princeton University, Princeton, NJ, in 2006 (under the supervision of A. Yao).

$\mathrm{He}$ is now holding a postdoctoral position at the California Institute of Technology, Pasadena.

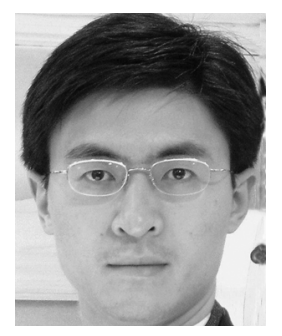

Mung Chiang (S'00-M'03) received the B.S (hons.) degree in electrical engineering and mathematics, and the M.S. and Ph.D. degrees in electrical engineering from Stanford University, Stanford, CA.

$\mathrm{He}$ is an Assistant Professor of Electrical Engineering and an affiliated faculty member of Applied and Computational Mathematics and of Computer Science at Princeton University, Princeton, NJ. He conducts research in the areas of optimization of communication systems, theoretical foundation of network architectures, algorithms in broadband access networks, and stochastic models of communications.

Prof. Chiang has been awarded as a Hertz Foundation Fellow, and received a Stanford University School of Engineering Terman Award, SBC Communications New Technology Introduction Contribution Award, NSF CAREER Award, and Princeton University Howard B. Wentz Junior Faculty Award. One of his papers becomes the Fast Breaking Paper in Computer Science in 2006 according to ISI's citation frequency. He also co-authored papers that received Best Student Paper Award at IEEE GLOBECOM. He is a co-editor of the new Springer book series on Optimization and Control of Communication Systems. He was the Lead Guest Editor of the Special Issue of IEEE JOURNAL OF SELECTED AREAS IN COMMUNICATIONS on "Nonlinear Optimization of Communication Systems," a Guest Editor of the Joint Special Issue of IEEE TRANSACTIONS ON INFORMATION THEORY and IEEE/ACM TRANSACTIONS ON NETWORKING on "Networking and Information Theory," an Editor of IEEE TRANSACTIONS ON WiRELESS COMMUNiCATIONS, a Program Co-Chair of the 38th Conference on Information Sciences and Systems. 